\title{
Quantitative spectroscopic analysis of and distance to SN1999em ${ }^{\star}$
}

\author{
L. Dessart ${ }^{1,3}$ and D. J. Hillier ${ }^{2}$ \\ 1 Max-Planck-Institut für Astrophysik, Karl-Schwarzschild-Str 1, 85748 Garching, Germany \\ 2 Department of Physics and Astronomy, University of Pittsburgh, 3941 O'Hara Street, Pittsburgh, PA 15260, USA \\ 3 Steward Observatory, University of Arizona, 933 North Cherry Avenue, Tucson, AZ 85721, USA \\ e-mail: luc@as.arizona.edu
}

Received 13 August 2005 / Accepted 17 October 2005

\section{ABSTRACT}

\begin{abstract}
Multi-epoch multi-wavelength spectroscopic observations of photospheric-phase type II supernovae (SN) provide information on massive-star progenitor properties, the core-collapse mechanism, and distances in the Universe. Following successes of recent endeavors (Dessart \& Hillier 2005a, A\&A, 437, 667; 2005b, A\&A, 439, 671) with the non-LTE model atmosphere code CMFGEN (Hillier \& Miller 1998, ApJ, 496, 407), we present a detailed quantitative spectroscopic analysis of the type II SN1999em and, using the Expanding Photosphere Method (EPM) or synthetic fits to observed spectra, à la Baron et al. (2004, ApJ, 616, 91), we estimate its distance. Selecting eight epochs, which cover the first 38 days after discovery, we obtain satisfactory fits to optical spectroscopic observations of SN1999em (including the UV and near-IR ranges when available). We use the same iron-group metal content for the ejecta, the same power-law density distribution (with exponent $n=10-12$ ), and a Hubble-velocity law at all times. We adopt a H/He/C/N/O abundance pattern compatible with CNO-cycle equilibrium values for a RSG/BSG progenitor, with $\mathrm{C} / \mathrm{O}$ enhanced and $\mathrm{N}$ depleted at later times. The overall evolution of the spectral energy distribution, whose peak shifts to longer wavelengths as time progresses, reflects the steady temperature/ionization-level decrease of the ejecta, associated non-linearly with a dramatic shift to ions with stronger line-blocking powers in the UV and optical (Fe II, TiII). In the parameter space investigated, CMFGEN is very sensitive and provides photospheric temperatures and velocities, reddenings, and the $\mathrm{H} / \mathrm{He}$ abundance ratio with an accuracy of $\pm 500 \mathrm{~K}$, $\pm 10 \%, 0.05$ and 50\%, respectively. Following Leonard et al. (2002, PASP, 114, 35), and their use of correction factors from Hamuy et al. (2001, ApJ, 558, 615), we estimate an EPM distance to SN1999em that also falls 30\% short of the Cepheid distance of $11.7 \mathrm{Mpc}$ to its host galaxy NGC 1637 (Leonard et al. 2003, ApJ, 594, 247). However, using the systematically higher correction factors of Dessart \& Hillier (2005b) removes the discrepancy. A significant scatter, arising primarily from errors in the correction factors and derived temperatures, is seen in distances derived using different band passes. However, adopting both correction factors and corresponding color-temperatures from tailored models to each observation leads to a good agreement between distance estimates obtained from different band passes. The need for detailed model computations thus defeats the appeal and simplicity of the original EPM method, which uses tabulated correction factors and broadband fluxes, for distance determinations. However, detailed fits to SN optical spectra, based on tailored models for individual SN observations, offers a promising approach to obtaining accurate distances, either through the EPM or via the technique of Baron et al. (2004). Our best distanceestimate to SN1999em is $11.5 \pm 1.0 \mathrm{Mpc}$. We note that to achieve $10-20 \%$ accuracy in such distance estimates requires multiple observations, covering preferentially a range of early epochs preceding the hydrogen-recombination phase.
\end{abstract}

Key words. radiative transfer - methods: numerical - stars: atmospheres - stars: supernovae: individual: SN1999em - stars: distances stars: evolution

\section{Introduction}

Stellar explosions, due to their large luminosity, represent an excellent probe of the distant, and thus young, Universe. Short Gamma Ray Bursts, identified with the death of Wolf-Rayet stars (MacFadyen \& Woosley 1999; MacFadyen et al. 2001), rank top of the prospective list, but currently, these phenomena are not suitably understood for distance calibration purposes. Down on the energetics ladder, supernovae explosions, discovered as bursts of optical light, have thus far been a viable route.

* Appendices are only available in electronic form at http://www . edpsciences.org
The inherent uniformity of Chandrasekhar-mass white-dwarfs, associated with type Ia SN, has motivated their nomination as standard candles. For the accuracy level sought, however, dust obscuration, metallicity effects, and (unknown) progenitor differences might invalidate such an assumption. Although typically ten times less luminous than type Ia, type II SN, resulting from the collapse of the Fe-core of a massive star, represent an alternative. By assuming their ejecta expand homologously and radiate a blackbody spectrum at a well-defined temperature, Kirshner \& Kwan (1974) designed the Expanding Photosphere Method (EPM), a variant of the Baade (1926) method for variable stars, to constrain the distance to a $\mathrm{SN}$. 
The agreement between the EPM-distance to SN1987A and that obtained, e.g., with LMC Cepheids, warranted application to a bigger sample, stretching into the Hubble flow and thus providing some independent constraint on the Hubble constant (Schmidt et al. 1992). But the 30-50\% lower value of the EPM-distance to SN1999em (Leonard et al. 2002, L02; Hamuy et al. 2001, H01) compared to the subsequently obtained Cepheid-distance to its host galaxy (NGC 1637; Leonard et al. 2003) led to question the reliability of the EPM. Using the Spectral-fitting Expanding Atmosphere Method (SEAM), Baron et al. (2004, B04) found a good agreement between their inferred distance to SN1999em and the Cepheid-distance to NGC 1637, and suggested that correction factors, used to approximate the Spectral Energy Distribution (SED) with that of a single-temperature blackbody, were too small at later epochs.

In this paper, we study the various ingredients entering the EPM to hunt for the origin of the above discrepancy, surprising because both methods share a number of assumptions or uncertainties: they both assume homologous expansion, are both affected by uncertainties in outflow expansion velocity or photometric measurements, and rely on multi-epoch observations to constrain the poorly-known time of explosion. Besides the generic $20 \%$ scatter of correction factors, the $20-50 \%$ lower values found by Eastman et al. (1996, E96) compared to those of Dessart \& Hillier (2005b, Paper II) could partly or fully explain the underestimate of the distance to NGC 1637 and SN1999em based on the EPM (H01, L02). Our approach to determining distances to type II SN requires a detailed modeling of multi-epoch spectroscopic observations; we thus also infer progenitor and $\mathrm{SN}$ outflow properties.

In Dessart \& Hillier (2005a, Paper I), we presented promising first analyses of photospheric-phase type II SN with CMFGEN (Hillier \& Miller 1998), a model atmosphere code so far devoted to studies of massive star winds (Najarro et al. 1997; Hillier \& Miller 1999; Dessart et al. 2000; Crowther et al. 2002; Martins et al. 2004). To step progressively through the various challenges posed by $\mathrm{SN}$ spectroscopic modeling, we first concentrate on type II SN, known to exhibit modest, and at early times negligible, amounts of nucleosynthesised metals during the explosion, thereby allowing us to neglect the deposition of energy from radioactive decay. Together with a Hubble velocity law, we implemented a simple power law density distribution $\rho(r)=\rho_{0}\left(R_{0} / r\right)^{n}$, where $\rho_{0}$ and $R_{0}$ are the base density and radius, and $n$ is the density exponent. Using spectroscopic observations of SN1987A and SN1999em, selected at times when hydrogen is partially or fully ionized, we found that both objects could be well modeled by adopting standard CNO-cycle equilibrium abundances typical of a supergiant massive star progenitor. Two optical features in early-time spectra, previously associated with peculiar line emission/absorption processes and/or outflow inhomogeneities lying high above the photosphere (Baron et al. 2000), were unambiguously identified as N II-multiplet lines. The strength of the features suggested a nitrogen enrichment, at the corresponding epoch, of a factor of a few compared to cosmic for SN1999em, but somewhat less for SN1987A. He I $5875 \AA$ could also be well reproduced with a modest helium enrichment, in contrast with former difficulties associated with that species (Eastman \& Kirshner 1989). Additionally, optical line profiles, and in particular their strong and persistent blueshift, were found to place a strong constraint on the density-gradient in the vicinity of the photosphere, requiring $n \sim 10$. For these test cases, CMFGEN fitted the UV, the optical and the near-IR (when available) with an accuracy generally better than a few tens of percent.

Overall, this high-level of agreement between our synthetic SEDs and spectroscopic observations of type II SN during their photospheric phase validates such a detailed approach with CMFGEN. It allows us to draw conclusions on the progenitor properties and perhaps more importantly on distances to the host galaxy.

In Dessart \& Hillier (2005b), we reviewed the various items entering the EPM, to complement the only other study of the kind done by Eastman et al. (1996, E96). CMFGEN yields correction factors that are systematically $\sim 20 \%$ larger than those of E96, but confirms the general properties with effectiveor blackbody color-temperature, and photospheric density. We also stressed that correction factors do not only correct for dilution by electron scattering of the SED set at the thermalization depth, but, within the standard domain of application of the EPM, also correct for the corrupting effects of line emission and absorption on the continuum flux within a given bandpass. Following the appearance of metal line blanketing below $T_{\text {phot }} \sim 8000 \mathrm{~K}$, such line effects dominate in the $B$-band (attributable to Fe II and Ti II) and in the I-band (Ca II), making the SED incompatible with that of a blackbody. Due to the steep increase of correction factors at low color-temperatures, modulations of line-strengths (having nothing to do with ionization but resulting from different metal abundances or expansion velocity) can affect inferred color-temperatures and generate substantial errors. Moreover, associated optical-depth enhancements in the corresponding spectral regions make the photosphere more extended than in the nearby line-free regions, invalidating the uniqueness of the photosphere radius required by the Baade method. These conclusions thus argue for the use of the EPM at early-times, when the outflow is fully ionized and metal-line blanketing confined to the UV region. Finally, although the velocity at maximum absorption $v_{\max }$ in optical P-Cygni profiles corresponds to the photospheric velocity $v_{\text {phot }}$ to within few tens of percents, we showed that $v_{\max }$ can over- as well as under-estimate $v_{\text {phot }}$, i.e. $v_{\max }$ does not necessarily exceed $v_{\text {phot }}$ (in absolute terms).

In this paper, we analyze a high-quality photometric and spectroscopic dataset for SN1999em with CMFGEN and infer its distance, first with the EPM in combination with various sources for correction factors, and then using CMFGEN fits to each epoch (an approach similar in spirit to the SEAM of B04). In Sect. 2, we present our selection of spectro-photometric observations, according to the criteria outlined above. In Sect. 3, we show the results of the quantitative spectroscopic modeling for each of the selected dates, drawing information on the outflow kinematics, chemistry, ionization. In Sect. 4, we compute the distance to SN1999em. In Sect. 4.1, using the standard minimization procedure and prescriptions for correction factors from $\mathrm{H} 01$, the EPM-distance shows a large scatter between selected band passes, is comparable to that found by L02 and $\mathrm{H} 01$ 
and thus always lower than the Cepheid-distance to NGC 1637. When we use the correction factors from Paper II, distance estimates are in better agreement with the Cepheid-distance, but there is still a relatively large scatter between different filter sets. A similar scatter occurs when we use blackbody color-temperatures extracted from the detailed models with the correction factors tabulated in Paper II. As expected, consistent distance estimates are obtained when we use the model blackbody color-temperatures together with the model correction factors. For comparison, we compute the SEAM-distance to SN1999em in Sect. 4.2 and corroborate the EPM-distance determination based on CMFGEN models or that of B04. In Sect. 5, we draw our conclusions and discuss future prospects.

\section{Spectrophotometric datasets of and reddening to SN1999em}

No neutrino detection was associated with the SN1999em event and thus no precise dating of the core-collapse of the progenitor exists. Inferences, based on the light curve appearance or the EPM/SEAM, suggest the explosion took place around the 25 th of October 1999, with an uncertainty of \pm 2 days (H01, L02, B04). It was absent in images of the host galaxy NGC 1637 taken on October 20.45 UT, while first detection of the SN was made on October 29th (JD 2451 480.94; Li 1999). The resemblance of the spectrum taken on October 30th with the spectrum of SN1987A taken on the 24th of February 1987, indicates that SN1999em was indeed caught at a very early phase of evolution.

In this work, we use two main sources of spectroscopic observations to model the evolution of SN1999em, from the first spectrum taken on the 30th of October 1999 until the last one taken on the 5th of December 1999, by which time metal lineblanketing in the optical spectral range is strong. From H01, we use optical spectra taken on the 30th of October, and the $3 \mathrm{rd}, 9 \mathrm{th}, 14 \mathrm{th}$, and 19th of November. In addition, we use the near-IR observations of $\mathrm{H} 01$ on the 2 nd and 19th of November. From the SUSPECT ${ }^{1}$ archive, we extract optical data of L02 for the 1 st and 5 th of November, and the 5th of December. Finally, we use HST-STIS observations taken on the 5th of November, kindly provided by Baron (priv. comm.), and covering the wavelength range from $1140 \AA$ to $5710 \AA$, overlapping in the red with observations of L02. On that date, we merge the two datasets to have a full coverage of the UV and optical out to $7500 \AA$. We exclude from our sample the observations of the 22nd and 24th of November (L02) because of dubious absolute flux level, curious relative flux distribution at and beyond $\mathrm{H} \alpha$ (compared to spectra taken at embracing dates; see discussion in L02). Hence, our sample contains eight observations for which photometric measurements have been obtained by L02, coincident to within a few hours to spectroscopic ones. On day 38, we adopt the magnitudes of L02 and combine these measurements with the spectrum taken on the previous day, i.e., the 5th of December, justified by the very slow spectroscopic evolution at such late times. In Table 1, we provide a $\log$ of the observations used in this work. Despite H01's

\footnotetext{
${ }^{1}$ www.bruford.nhn.ou.edu/ suspect
}

Table 1. Log of the observations used and described in Sect. 2. Photometric measurements, with corresponding dates, are reproduced from L02 (Cols. 2-5). Spectroscopic datasets are referenced according to spectral range, i.e. UV, optical (Opt.) and near-IR (nIR). Note that in Sect. 4, the photometric data on day 38 is combined with the spectrocopic data on day 37 .

\begin{tabular}{lccccccc}
\hline \hline Date & Day $^{a}$ & \multicolumn{3}{c}{ Photometry $^{b}$} & \multicolumn{3}{c}{ Spectroscopy } \\
(1999) & & $B$ & $V$ & $I$ & UV & Opt. & nIR \\
\hline 30 Oct. & 1.0 & 13.87 & 13.87 & 13.65 & $\ldots$ & 1 & $\ldots$ \\
01 Nov. & 3.0 & 13.80 & 13.79 & 13.56 & $\ldots$ & 2 & $\ldots$ \\
02 Nov. & 4.0 & $\ldots$ & $\ldots$ & $\ldots$ & $\ldots$ & $\ldots$ & 1 \\
03 Nov. & 5.0 & 13.85 & 13.79 & 13.54 & $\ldots$ & 1 & $\ldots$ \\
05 Nov. & 7.0 & 13.92 & 13.84 & 13.53 & 3 & 2,3 & $\ldots$ \\
09 Nov. & 11.0 & 14.02 & 13.84 & 13.48 & $\ldots$ & 1 & $\ldots$ \\
14 Nov. & 16.0 & 14.25 & 13.81 & 13.44 & $\ldots$ & 1 & $\ldots$ \\
19 Nov. & 21.0 & 14.47 & 13.86 & 13.40 & $\ldots$ & 1 & 1 \\
06 Dec. & 38.0 & 14.94 & 13.93 & 13.29 & $\ldots$ & 2 & $\ldots$ \\
\hline
\end{tabular}

${ }^{a}$ Days since JD $2451480.94 ;{ }^{b}$ measurements are taken from 1: H01; 2: L02; 3: Baron et al. (2000).

photometric measurements over a large time-span in the $Z, J$, $H$, and $K$ bands, we limit our investigation to the optical band passes $B, V$, and $I$, and sets $\{B, V\},\{B, V, I\}$, and $\{V, I\}$ (note one exception with the $Z$-band in Sect. 4.2; see H01 for a presentation of this $Z$-band).

Our modeling procedure blueshifts the spectroscopic observations of SN1999em by $770 \mathrm{~km} \mathrm{~s}^{-1}$ (L02), scales the synthetic flux to the Cepheid-distance of $11.7 \mathrm{Mpc}$ (L03), and finally reddens such a scaled synthetic spectrum using the Cardelli et al. (1998) law, adopting $A_{V} / E(B-V)=3.1$. In Paper I, we illustrated the mingled effects on the SED associated with metal line-blanketing, cooling of the outflow and reddening. Here, the fine time-sampling and the HST-STIS data can only be fitted with CMFGEN by adopting a low reddening value of $0.1 \pm 0.05$, in agreement with previous studies (L02, Baron et al. 2000, B04; see also Sect. 3.3.2). Note that the UV data is indeed a key asset for reddening determinations since the SED at longer wavelength becomes less and less sensitive to reddening modulations. In the context of the EPM, the extinction uncertainty translates directly into an uncertainty in the distance modulus. Fortunately, due to the low ISM column density to $\mathrm{SN} 1999 \mathrm{em}$, this introduces modest absolute errors on the distance (see Sect. 4.2).

To highlight line-emission contributions, we always plot the synthetic spectrum obtained by including all opacity sources (red curve) and that accounting only for continuum processes (blue curve). Finally, the adopted model luminosities for each date match the absolute observed flux level (for the assumed Cepheid-distance) to within a factor of approximately 2-3 (our initial adoption of the lower distance estimate by H01/L02 when modelling SN1999em introduced a systematic underestimate of the luminosities by a factor of $\left.\sim(11.7 / 8)^{2} \sim 2.1\right)$; the synthetic SED shows only a modest sensitivity to luminosity scalings of that magnitude, provided the outflow ionization is preserved (Paper I, E96). 
In Sects. 4.1-4.2, we convert synthetic and blackbody fluxes into magnitudes using the filter transmission functions and zero-points of $\mathrm{H} 01$ and Hamuy (priv. comm.), adopting either the value at each filter's effective wavelength $\left(A_{B}=\right.$ $4.05 E(B-V)$ and $\left.A_{I}=1.83 E(B-V)\right)$, or convolving the synthetic SED with the filter transmission function. Resulting magnitudes from the two approaches differ by no more than 0.005 mag, thus much less than other uncertainties involved in the EPM or SEAM.

\section{Quantitative spectroscopic analysis of SN1999em}

In this section, we perform a quantitative spectroscopic analysis of SN1999em, at eight dates sampling its early photospheric-phase evolution (see above). In Sect. 3.1, we review the standard model parameters and assumptions. We then detail our results for each observation in Sect. 3.2. Finally, in Sect. 3.3, we discuss typical uncertainties associated with each of the inferred model parameters.

\subsection{Model presentation}

The standard set of parameters needed by CMFGEN were discussed thoroughly in Paper I. A key asset of CMFGEN is the explicit treatment of line-blanketing, justifying a detailed description of the atomic structure for a large number of species. Models discussed below include H I $(30,20)$, He I (51, 40), C I (63, 33), C II (59, 32), C III (20, 12), C IV (14, 9), N I (104, 44), N II $(41,23)$, N III $(8,8)$, O I $(75,23)$, O II $(111,30)$, O III $(46,26)$, Ne II $(242,42)$, Na I $(71,22)$, Na II $(35,21), \operatorname{Mg}$ II $(65,22)$, Al II $(44,26)$, Al III (45, 17), Si II $(59,31)$, Si III $(51,27)$, S II $(324,56)$, S III $(98,48)$, Ca II $(77,21)$, Ti II $(152,37)$, Ti III $(206,33)$, Cr II $(196,28)$, Cr III $(145,30)$, Mn II $(97,25)$, Mn III $(175,30)$, Fe I $(136,44)$, Fe II $(309,116)$, Fe III $(477,61)$, Fe IV $(282,50)$, Co II $(144,34)$, Co III $(283,41)$, Ni II $(93,19)$, Ni III $(67,15)$, each parenthesis containing the number of full- and superlevels (see Hillier \& Miller 1998, for details). At epochs when a given species offers a negligible opacity to the radiation field at all depths in the outflow, we exclude it from the atomic dataset, thereby reducing the memory requirements and accelerating the convergence of CMFGEN. Under typical type II SN conditions and when all the above species are included, CMFGEN uses up to $3.5 \mathrm{~GB}$ of RAM and may take a week to reduce all level population fluctuations, from one iteration to the next, to less than $1 \%$.

As discussed below, the main component controlling the evolution of type II SN spectra during their photospheric phase is the steady decrease of the outflow temperature, in parallel with the reduction of the outflow ionization. As time proceeds, opacity sources change, and the opacity may increase (e.g., when Fe II dominates over Fe III). In contrast to the large changes in effective temperature, we find only small and specific changes in chemical composition as time progresses through the first 40 days past discovery. No metal-abundance variations associated with explosive nucleosynthesis are found, so that all spectra are fitted with a unique metallicity, adopted to be solar (L02). For hydrogen and helium, we reproduce all epochs with a unique value, $\mathrm{H} / \mathrm{He}=5$ (by number), although our inability to fit the Balmer lines at late times makes our result reliable only up to the hydrogen-recombination phase (first twenty days). The nitrogen abundance is constrained from the $\mathrm{N}$ II optical lines (Paper I) and requires over-solar abundance; we adopt $\mathrm{N} / \mathrm{He}=6.8 \times 10^{-3}$ (Prantzos et al. 1986). $\mathrm{C}$ and $\mathrm{O}$ abundances are difficult to constrain: at early times, since no lines from these species are observed, we adopt $\mathrm{C}$ and $\mathrm{O}$ abundances compatible with the $\mathrm{N} / \mathrm{He}$ above and $\mathrm{CNO}$-cycle equilibrium values, with $\mathrm{C} / \mathrm{He}=1.7 \times 10^{-4}$ and $\mathrm{O} / \mathrm{He}=10^{-4}$ by number. At later times, however, $\mathrm{C} I$ and $\mathrm{O} I$ lines are unambiguously observed in the 7000-12000 $\mathrm{A}$ region, requiring enhancements compared to $\mathrm{CNO}$ equilibrium values, of a factor of 10 for carbon and 100 for oxygen (Sect. 3.2.8). Finally, the NaI5890 $\AA$ doublet can only be fitted with a factor of four over-abundance compared to solar, in agreement with the CNO-cycle equilibrium values of Prantzos et al. (1986), i.e. $X_{\mathrm{Na}}=1.38 \times 10^{-4}$. Overall, we note that the conspicuous spectroscopic evolution during the photospheric phase is not a reflection of outflow changes in chemical composition but rather in ionization/temperature.

The density distribution of the outflow is constrained via the density exponent $n$. In Papers I and II, we illustrated, from different perspectives, the spatial confinement of line and continuum formation regions to a few density scale heights about the photosphere rather than over the entire outflow - the Ca II set of lines at around $8500 \AA$ is a noticeable exception (e.g., Fig. 13., Paper I). The resulting line-profile shapes require values $n \gtrsim 10$ to reproduce the blueshift as well as both the modest absorption and emission observed in Balmer lines. Comparable values are obtained in other spectroscopic studies, e.g. Eastman \& Kirshner (1989), or independent radiationhydrodynamical simulations of core-collapse (Arnett 1988; Woosley 1988; Ensman \& Burrows 1992).

We quote numerous model properties at the photosphere, which corresponds here to the location where the continuum optical depth, integrated inwards from the outer grid radius, is $2 / 3$. We constrain the expansion, or, equivalently, the photospheric velocity by adjusting the velocity at the inner boundary; we then adopt the value that permits a satisfactory fit to profile shapes for a wide range of optical line diagnostics, a procedure we favor over the direct and more precise, but less meaningful, measurements on individual observed line profiles. Further discussion on this is provided in Sect. 5 of Paper II, as well as below, in Sect. 3.3.

\subsection{Tailored analysis with CMFGEN}

In this section we model the data for our selected observation dates (Sect. 2), provide synthetic fits to observed SEDs (Figs. 1-8), and tabulate the derived model parameters (Table 2). As described in the preceding section, the outflow temperature, modulated via the base luminosity or radius, represents the essential change between consecutive dates. The electron temperature at the photosphere, $T_{\text {phot }}$, accounts well for this change, and thus constitutes the center of the 
Table 2. Summary of model results presented in Sect. 3.2. Time reference is in days since JD 2451480.94 . On the right hand side, we also give the key EPM quantities for each model and date, i.e., blackbody color temperature and corresponding correction factor for each bandpass, $\{B, V\},\{B, V, I\}$, and $\{V, I\}$ (Sect. 4; Paper II).

\begin{tabular}{ccccccccccccc}
\hline \hline Day & $\begin{array}{c}L_{*} \\
\left(10^{8} L_{\odot}\right)\end{array}$ & $\begin{array}{c}T_{\text {phot }} \\
(\mathrm{kK})\end{array}$ & $\begin{array}{c}R_{\text {phot }} \\
\left(10^{14} \mathrm{~cm}\right)\end{array}$ & $\begin{array}{c}v_{\text {phot }} \\
\left(\mathrm{km} \mathrm{s}^{-1}\right)\end{array}$ & $\begin{array}{c}\rho_{\text {phot }} \\
\left(10^{-14} \mathrm{~g} \mathrm{~cm}^{-3}\right)\end{array}$ & $\begin{array}{c}T_{B V} \\
(\mathrm{kK})\end{array}$ & $\xi_{B V}$ & $\begin{array}{c}T_{B V I} \\
(\mathrm{kK})\end{array}$ & $\xi_{B V I}$ & $\begin{array}{c}T_{V I} \\
(\mathrm{kK})\end{array}$ & $\xi_{V I}$ \\
\hline 1.0 & 15.0 & 13.38 & 6.2 & 10430 & 4.3 & 12 & 16.02 & 0.462 & 14.96 & 0.50 & 14.39 & 0.52 \\
3.0 & 15.0 & 12.59 & 6.8 & 8940 & 3.3 & 10 & 15.96 & 0.430 & 15.03 & 0.46 & 14.23 & 0.49 \\
4.0 & 15.0 & 12.59 & 6.8 & 8940 & 3.3 & 10 & 15.96 & 0.430 & 15.03 & 0.46 & 14.23 & 0.49 \\
5.0 & 9.0 & 10.63 & 6.7 & 8840 & 3.6 & 10 & 14.23 & 0.443 & 13.20 & 0.49 & 12.53 & 0.51 \\
7.0 & 5.0 & 9.20 & 6.64 & 8750 & 4.1 & 10 & 12.95 & 0.443 & 11.95 & 0.49 & 11.28 & 0.53 \\
11.0 & 2.5 & 8.04 & 6.65 & 7960 & 4.0 & 10 & 10.70 & 0.469 & 10.25 & 0.50 & 9.83 & 0.53 \\
16.0 & 1.5 & 6.80 & 6.15 & 6350 & 8.7 & 10 & 8.17 & 0.646 & 8.84 & 0.56 & 9.55 & 0.51 \\
21.0 & 3.75 & 6.26 & 12.90 & 5530 & 7.4 & 10 & 7.11 & 0.685 & 7.85 & 0.56 & 8.74 & 0.48 \\
38.0 & 2.0 & 5.92 & 10.90 & 3400 & 38.8 & 10 & 5.54 & 1.072 & 6.34 & 0.75 & 7.24 & 0.61 \\
\hline
\end{tabular}

discussion below. To infer the photospheric velocity, many models were calculated: we adopted the photospheric velocity corresponding to that model which provided the best fit to the majority of observed line profiles. A detailed discussion on the uncertainties in the derived photospheric velocity is provided in Sect. 3.3.3.

\subsubsection{Observation of the 30th of October 1999}

In the top panel of Fig. 1, we present a fit of the reddened scaled synthetic spectrum (full: thick red; continuum: thin blue) to the observations taken on the 30th of October 1999 (black; H01). The model parameters are: $L_{*}=1.5 \times 10^{9} L_{\odot}$, $T_{\text {phot }}=13380 \mathrm{~K}, R_{\text {phot }}=6.2 \times 10^{14} \mathrm{~cm}, v_{\text {phot }}=10430 \mathrm{~km} \mathrm{~s}^{-1}$, $\rho_{\text {phot }}=4.3 \times 10^{-14} \mathrm{~g} \mathrm{~cm}^{-3}$, and $n=12$. The flux, underestimated by $17 \%$, is renormalised at $8200 \AA$. The overall fit quality is good, although the flux around $3800 \AA$ is underestimated. Including more species or bigger model atoms makes no difference; increasing $T_{\text {phot }}$ helps but then provides a poor match to the observed flux elsewhere, e.g., below $3500 \AA$. A poor flux calibration in this spectral region could be at the origin of this discrepancy. The fit quality to Balmer lines is satisfactory, although the extent of profile troughs is underestimated. At such early times, our neglect of relativistic terms in the radiative transfer equation may contribute to such discrepancies (Hauschildt et al. 1991; Jeffery 1993). Helium lines, and most notably He I $5875 \AA$, are well reproduced. In the bottom panel of Fig. 1, we show synthetic spectrum fits (thin colored-lines) to observations, but treating chemical species in isolation. As emphasized in Paper I, we predict N II (orange line) features in the blue wing of He I $5875 \AA$, resulting from the $3 d-3 p$ multiplet around $5470 \AA$ and the $3 \mathrm{p}-3$ s multiplet around $5670 \AA$, as well as in the blue wing of $\mathrm{H} \beta$, resulting from other $\mathrm{N}$ II multiplets around $4600 \AA$. Finally, notice how the continuum lies in general well below the full synthetic flux distribution level, even in spectral regions that look essentially featureless, revealing the ubiquitous presence of line emission (see, e.g., the subtle contribution from Fe III - colored in red - to the red wing of both $\mathrm{H} \beta$ and He I $5875 \AA$, or that of He I $6678 \AA$ in the red wing of $\mathrm{H} \alpha$ ).

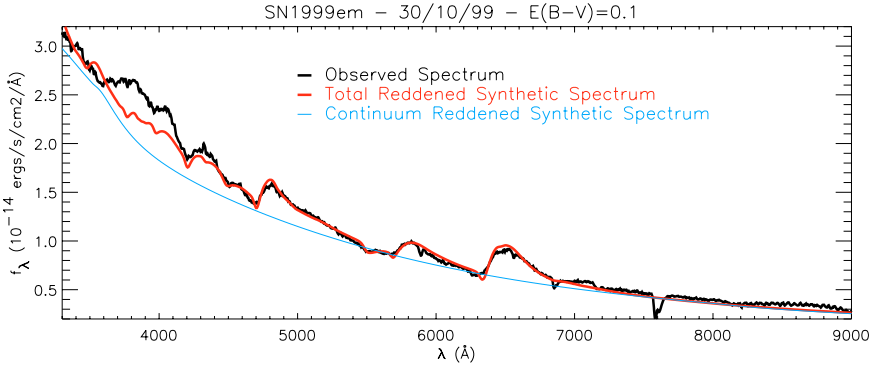

$S N 1999 e m-30 / 10 / 99-E(B-V)=0.1$

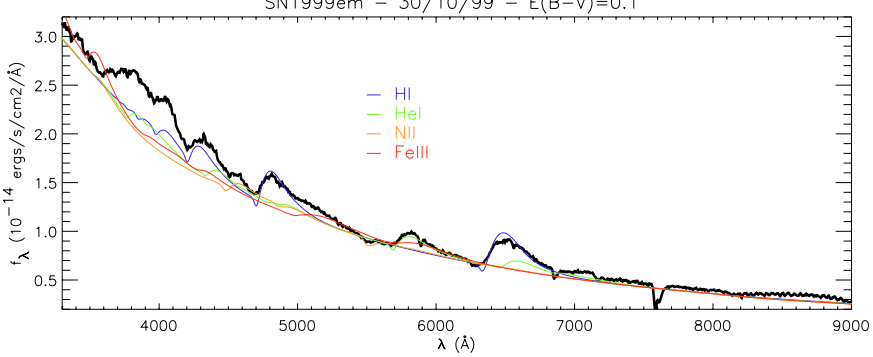

Fig. 1. Top: fit of the full (red) and continuum (blue) synthetic spectra to observations (black) of SN1999em taken on the 30th of October 1999 (Day 1; H01). Model parameters are: $L_{*}=1.5 \times 10^{9} L_{\odot}$, $T_{\text {phot }}=13380 \mathrm{~K}, R_{\text {phot }}=6.2 \times 10^{14} \mathrm{~cm}, v_{\text {phot }}=10430 \mathrm{~km} \mathrm{~s}^{-1}$, $\rho_{\text {phot }}=4.3 \times 10^{-14} \mathrm{~g} \mathrm{~cm}^{-3}$, and $n=12$. The synthetic flux, $17 \%$ lower than observed, is re-normalized at $8200 \AA$ A. Bottom: same as above, but this time overplotting reddened synthetic spectra computed by treating bound-bound transitions of a single species: $\mathrm{HI}, \mathrm{He}$ I, N II, and Fe III. Contrary to Baron et al. (2000), we attribute the two features just blueward of $\mathrm{H} \beta$ and He I $5875 \AA$ to N II lines. (This figure is available in color in the electronic version.)

\subsubsection{Observations of the 1st-2nd of November 1999}

In Fig. 2, we reproduce Fig. 1 for the observations of the 1 st (optical, top panel; L02) and the 2nd (near-IR, bottom panel; H01) of November 1999. The full (thick red) and continuum (thin blue) synthetic flux distributions underestimate by $30 \%$ the observed flux and are thus re-normalized, at $6900 \AA$, to fit both spectral ranges (with an additional $8 \%$ flux adjustment between optical and near-IR). The model parameters, identical for both spectral ranges, are: $L_{*}=1.5 \times 10^{9} L_{\odot}$, 

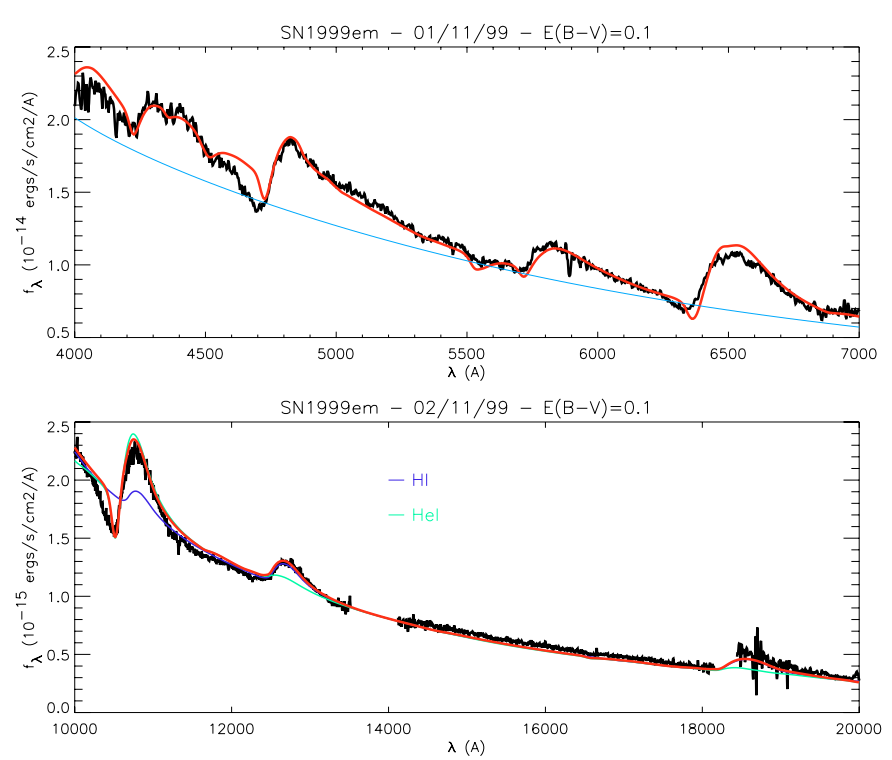

Fig. 2. Fit of the full (red) and continuum (blue) synthetic spectra to observations (black) of SN1999em taken on the 1st (optical, L02, day 3; top) and the 2nd (near-IR, H01, day 4; bottom) of November 1999. In the bottom panel, we include the synthetic spectra obtained by restricting bound-bound transitions to those of $\mathrm{HI}$ (blue) or He I (green). The model parameters are: $L_{*}=1.5 \times 10^{9} L_{\odot}$, $T_{\text {phot }}=12590 \mathrm{~K}, R_{\text {phot }}=6.8 \times 10^{14} \mathrm{~cm}, v_{\text {phot }}=8940 \mathrm{~km} \mathrm{~s}^{-1}, \rho_{\text {phot }}=$ $3.3 \times 10^{-14} \mathrm{~g} \mathrm{~cm}^{-3}$ and $n=10$. The model flux, which underestimates the observations by $30 \%$, is renormalized to the observed value at $6900 \AA$. (This figure is available in color in the electronic version.)

$T_{\text {phot }}=12590 \mathrm{~K}, R_{\text {phot }}=6.8 \times 10^{14} \mathrm{~cm}, v_{\text {phot }}=8940 \mathrm{~km} \mathrm{~s}^{-1}$, $\rho_{\text {phot }}=3.3 \times 10^{-14} \mathrm{~g} \mathrm{~cm}^{-3}$, and $n=10$.

In the optical, there is little change with observations taken on the 30th of October, reflected in the similarity of model parameters for both dates. We obtain good fits to Balmer as well as Paschen lines, i.e., $\mathrm{P} \gamma 1.094 \mu \mathrm{m}, \mathrm{P} \beta 1.28 \mu \mathrm{m}$, and $\mathrm{P} \alpha 1.875 \mu \mathrm{m}$. Note, however, that profile troughs are sometimes predicted too narrow (e.g., $\mathrm{H} \alpha$ and $\mathrm{H} \beta$ ). He I lines are also well reproduced, both in the optical at $5875 \AA$ and in the near-IR at $1.083 \mu \mathrm{m}$ (which overlaps with $\mathrm{P} \gamma$ but contributes most of the flux seen at this date in the $1.08 \mu \mathrm{m}$ feature). Note the broad N II feature blueward of He I $5875 \AA$, although its absorption strength is somewhat overestimated (see Sect. 3.3).

\subsubsection{Observation of the 3rd of November 1999}

In Fig. 3, we present a fit of the full (red) and continuum (blue) synthetic SED to the observed SED of the 3rd of November 1999 (black; H01). The model has the following properties: $L_{*}=9 \times 10^{8} L_{\odot}, T_{\text {phot }}=10630 \mathrm{~K}, R_{\text {phot }}=6.7 \times 10^{14} \mathrm{~cm}$, $v_{\text {phot }}=8840 \mathrm{~km} \mathrm{~s}^{-1}, \rho_{\text {phot }}=3.6 \times 10^{-14} \mathrm{~g} \mathrm{~cm}^{-3}$, and $n=10$. The model flux, underestimated by $50 \%$, is renormalised to the observed value at $5400 \AA$. The fit quality is good. Increasing the outflow temperature (or ionization) results in a better fit of the overall shape of the spectrum but leads to an overestimate of the strength of He I $5875 \AA$ and that of the N II $5400 \AA$ feature. The bump in the red wing of $\mathrm{H} \beta$, due primarily to Fe III lines, is underestimated, suggesting perhaps a slightly higher environmental metallicity (Paper I). As noted in the preamble to the

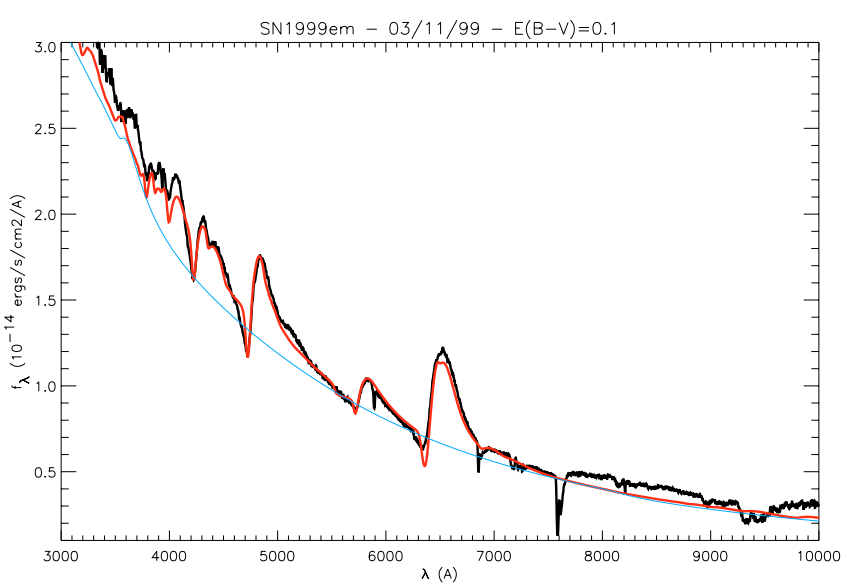

Fig. 3. Synthetic fits (color) to observations (black) taken on November 3rd (H01; day 5). The model has the following parameters: $L_{*}=9 \times 10^{8} L_{\odot}, T_{\text {phot }}=10630 \mathrm{~K}, R_{\text {phot }}=6.7 \times 10^{14} \mathrm{~cm}$, $v_{\text {phot }}=8840 \mathrm{~km} \mathrm{~s}^{-1}, \rho_{\text {phot }}=3.6 \times 10^{-14} \mathrm{~g} \mathrm{~cm}^{-3}$, and $n=10$. The model flux, which underestimates the observations by $50 \%$, is renormalized to the observed value at $5400 \AA$. (This figure is available in color in the electronic version.)

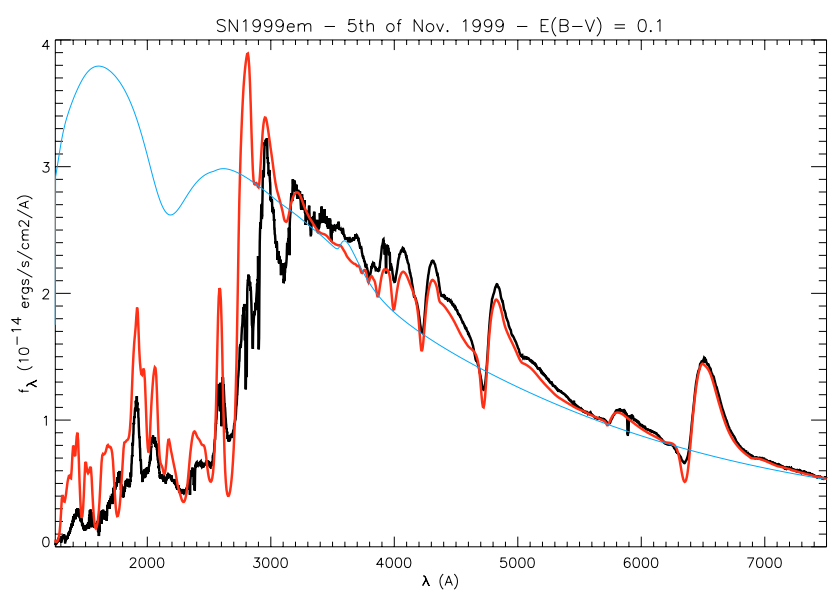

Fig. 4. Full (red) and continuum (blue) synthetic fits to observations (black) taken on November 5th (UV range: Baron et al. 2000; optical range: L02, day 7). The model has the following parameters: $L_{*}=$ $5 \times 10^{8} L_{\odot}, T_{\text {phot }}=9200 \mathrm{~K}, R_{\text {phot }}=6.64 \times 10^{14} \mathrm{~cm}, v_{\text {phot }}=8750 \mathrm{~km} \mathrm{~s}^{-1}$, $\rho_{\text {phot }}=4.1 \times 10^{-14} \mathrm{~g} \mathrm{~cm}^{-3}$, and $n=10$. The model flux, which underestimates the observations by a factor of two, is renormalized to the observed value at $7500 \AA$. The dip in the continuum spectrum near $2200 \AA$ is due to the effects of interstellar extinction. (This figure is available in color in the electronic version.)

section, we use, by default, a solar mixture of metals. Finally, note how reduced the continuum flux level is compared to the full SED, highlighting the ubiquitous presence of broad and weak "background" lines.

\subsubsection{Observation of the 5th of November 1999}

In Fig. 4, we show a full (red) and continuum (blue) synthetic fit to observations taken on the 5th of November 1999 (L02), the optical range being complemented with UV HST data (Baron et al. 2000; see Sect. 2). The model has the following parameters: $L_{*}=5 \times 10^{8} L_{\odot}, T_{\text {phot }}=9200 \mathrm{~K}, R_{\text {phot }}=6.64 \times 10^{14} \mathrm{~cm}$, 


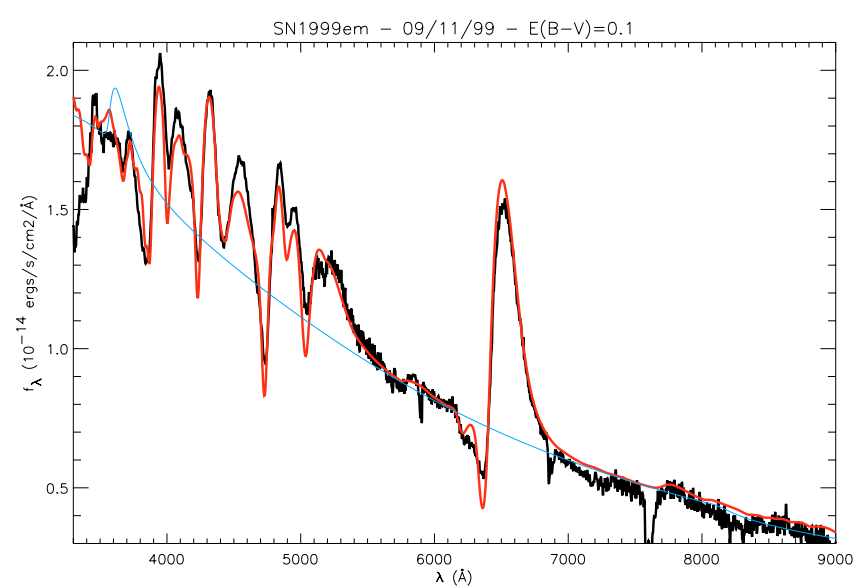

Fig. 5. Synthetic fits (red) to observations (black) taken on November 9th (H01; day 11). The model has the following parameters: $L_{*}=2.5 \times$ $10^{8} L_{\odot}, T_{\text {phot }}=8040 \mathrm{~K}, R_{\text {phot }}=6.65 \times 10^{14} \mathrm{~cm}, v_{\text {phot }}=7960 \mathrm{~km} \mathrm{~s}^{-1}$, $\rho_{\text {phot }}=4 \times 10^{-14} \mathrm{~g} \mathrm{~cm}^{-3}$, and $n=10$. The model flux, which underestimates the observations by a factor of 2.65 , is renormalized to the observed value at $6000 \AA$. The feature in the continuum spectrum near $3600 \AA$ arises from the inclusion, via level dissolution, of Balmer series bound-bound transitions near the series limit. (This figure is available in color in the electronic version.)

$v_{\text {phot }}=8750 \mathrm{~km} \mathrm{~s}^{-1}, \rho_{\text {phot }}=4.1 \times 10^{-14} \mathrm{~g} \mathrm{~cm}^{-3}$, and $n=10$. The model flux, which underestimates the observations by a factor of two, is renormalized to the observed value at $7500 \AA$. Both observation and model were discussed in detail in Paper I (Sect. 3.2), emphasizing the complex and primary role played by metal line-blanketing in the UV range (see lower panel of Fig. 3, Paper I).

The fit quality is good but there are noticeable discrepancies. The optical flux is somewhat underestimated, while the UV flux is overestimated in a number of wavelength regions. We overestimate the flux in the $2800 \AA$ region, which, additionally to Fe II, is strongly influenced by the multiplet of $\mathrm{Mg}$ II, composed of $\mathrm{Mg}$ II (3p-3s) and $\mathrm{Mg}$ II (4s-3p). Increasing/decreasing the Magnesium abundance by a factor of two introduces flux changes only at the $10 \%$ level, thus insufficient to resolve the discrepancy. In Paper I, we showed how a global increase of the pristine metal abundance leads to a better match, e.g., of the strength of the iron feature at $\sim 5000 \AA$, giving some support for a solar or over-solar metallicity of the SN1999em environment; simultaneously, the increased metalline blanketing in the UV reduces the emergent UV flux to more suitable levels. Alternatively, lowering the effective temperature increases line-blocking through the shift from Fe III to Fe II opacity sources, also matching the observed UV flux better; in this situation, however, the slope of the optical synthetic SED is then too flat. We discuss further these issues in Sect. 3.3 but already here, we see how coupled and subtle the effects of line-blanketing and outflow temperature/ionization are. Note that the photospheric temperature of $9200 \mathrm{~K}$ found here is in good agreement with the value of $9000 \mathrm{~K}$ proposed for that date by Baron et al. (2000).

Overall, having access to UV data during the early evolution is an asset, as it allows the determination, or the

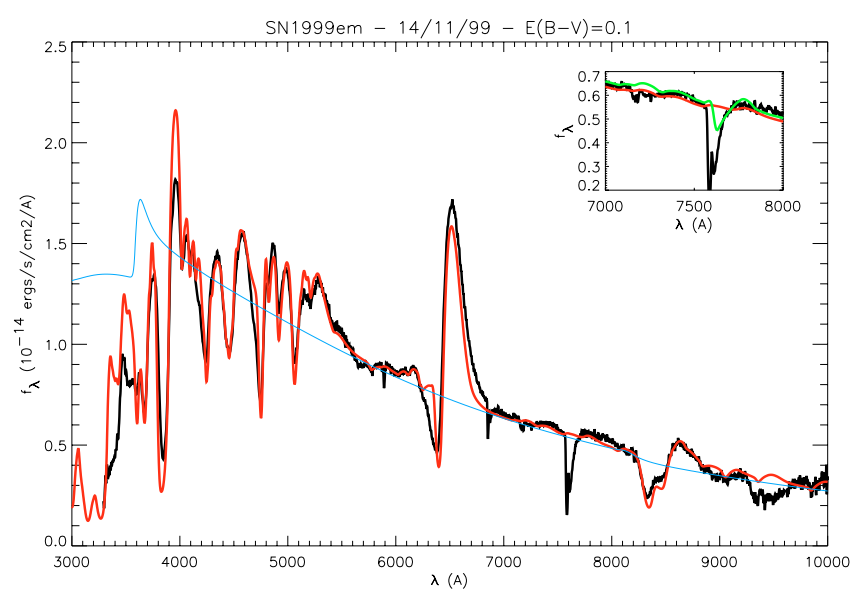

Fig. 6. Full (red) and continuum (blue) synthetic spectral fits to observations (black) taken on November 14th (H01; day 16). The model has the following parameters: $L_{*}=1.5 \times 10^{8} L_{\odot}, T_{\text {phot }}=6800 \mathrm{~K}$, $R_{\text {phot }}=6.15 \times 10^{14} \mathrm{~cm}, v_{\text {phot }}=6350 \mathrm{~km} \mathrm{~s}^{-1}, \rho_{\text {phot }}=8.7 \times 10^{-14} \mathrm{~g} \mathrm{~cm}^{-3}$ and $n=10$. The model flux, which underestimates the observations by a factor of 3.7 , is renormalized to the observed value at $6000 \AA$. The species identification of line contributions is shown in the lower panel of Fig. 5, Paper I. (This figure is available in color in the electronic version.)

confirmation, of the reddening obtained usually at other (relatively late) times from optical data.

\subsubsection{Observation of the 9th of November 1999}

We show in Fig. 5 a synthetic fit to the observations of SN1999em on the 9th of November 1999 (H01; day 11). The model parameters are the following: $L_{*}=2.5 \times 10^{8} L_{\odot}$, $T_{\text {phot }}=8040 \mathrm{~K}, R_{\text {phot }}=6.65 \times 10^{14} \mathrm{~cm}, v_{\text {phot }}=7960 \mathrm{~km} \mathrm{~s}^{-1}$, $\rho_{\text {phot }}=4 \times 10^{-14} \mathrm{~g} \mathrm{~cm}^{-3}$, and $n=10$. The model flux, which underestimates the observations by a factor of 2.65 , is renormalized to the observed value at $6000 \AA$. Starting around this date, metal line blanketing in the optical is noticeable, increasing steadily as we progress through the photospheric phase. Mingled with the Balmer lines $(\mathrm{H} \gamma, \mathrm{H} \beta$, and $\mathrm{H} \alpha)$, one sees the presence of Ca II at $3800 \AA$ (as for the previous date), Fe II and Ti II lines at $\sim 4400 \AA$, and Fe II lines redward of $\mathrm{H} \beta$ up to $5500 \AA$. He I $5875 \AA$ is still observable as a very weak feature, soon to be replaced at the same location by the NaI5890 doublet. Fe II and Si II are also present around $6300 \AA$, creating a kink in the $\mathrm{H} \alpha$ absorption trough; it might thus not be necessary to invoke a peculiar formation of the $\mathrm{H} \alpha$ line to explain this feature (L02).

\subsubsection{Observation of the 14th of November 1999}

In Fig. 6, we present full (red) and continuum (blue) synthetic spectrum fits to observations taken on the 14th of November 1999 (H01; day 16). The model parameters are the following: $L_{*}=1.5 \times 10^{8} L_{\odot}, T_{\text {phot }}=6800 \mathrm{~K}, R_{\text {phot }}=6.15 \times 10^{14} \mathrm{~cm}$, $v_{\text {phot }}=6350 \mathrm{~km} \mathrm{~s}^{-1}, \rho_{\text {phot }}=8.7 \times 10^{-14} \mathrm{~g} \mathrm{~cm}^{-3}$, and $n=10$. The model flux, which underestimates the observations by a factor of 3.7, is renormalized to the observed value at $6000 \AA$. 
Note that a detailed discussion of this model has been made in Sect. 3.3 of Paper I.

A conspicuous feature now appears in the $I$ band, attributable to Ca II 8498-8542-8662 $\AA$, consistent with the identification of Ca II 3933-3968 $\AA$ at earlier epochs. He I $5875 \AA$ has also vanished, replaced by a weak feature due to Na I $5890 \AA$ A, well fitted by invoking an enhancement of four over the corresponding solar sodium abundance (Paper I; Prantzos et al. 1986). The feature at $7700 \AA$ now seems to extend too far to the red to be attributed exclusively to terrestrial atmospheric absorption, as supposed so far, and thus suggests the presence of OI $7700 \AA$. In the top right corner of Fig. 6 , we add, over the relevant region, the synthetic SED (green) obtained by enhancing the oxygen abundance by a factor of 100 over the standard value $\mathrm{O} / \mathrm{He}=10^{-4}$, as required to reproduce the red-side of the observed feature. Observations at later epochs, revealing spectra with features both narrower and better centered on the rest-wavelength (see Paper I), will confirm this identification and the need for such a significant enhancement of the oxygen abundance over the CNO-cycle equilibrium value, characteristic of the deeper layers of the progenitor star (Hirshi et al. 2004). Metal line features in the optical have also strengthened compared to those of the previous date, following the cooling of the outflow, and the recombination of hydrogen and Fe III (to Fe II). The $\mathrm{H} \alpha$ line profile is not too well fitted: its red-wing extent is underestimated as is the P-Cygni trough, which is now very pronounced. We instead predict a narrower profile shape, with considerable filling-in of the trough by Fe II and Si II lines. As we will see below, this problem only gets worse, associated with the infamous underestimate of the $\mathrm{H} \alpha$ strength.

\subsubsection{Observation of the 19th of November 1999}

We show in Fig. 7 synthetic fits (color) to the observations of the 19th of November 1999 (H01; day 21). The model flux is normalized to the observed one at $6100 \AA$ with an additional decrease of $15 \%$ in the near-IR. This flux shift is likely to result from the time difference between the two observations rather than an inadequate slope between the optical and the near-IR ranges in the synthetic spectrum. The model properties are the following: $L_{*}=3.75 \times 10^{8} L_{\odot}, T_{\text {phot }}=6260 \mathrm{~K}, R_{\text {phot }}=12.9 \times$ $10^{14} \mathrm{~cm}, v_{\text {phot }}=5530 \mathrm{~km} \mathrm{~s}^{-1}, \rho_{\text {phot }}=7.4 \times 10^{-14} \mathrm{~g} \mathrm{~cm}^{-3}$, and $n=10$. The model flux, which underestimates the observations by $10 \%$, is renormalised to the observed value at $6100 \AA$.

As described in Paper I, we have found that using a small turbulent velocity helps maintain significant emission in $\mathrm{H} \alpha$ (see next section). In general, this entails using a turbulent velocity of $20 \mathrm{~km} \mathrm{~s}^{-1}$, rather than $100 \mathrm{~km} \mathrm{~s}^{-1}$ used for models where hydrogen is fully or mostly ionized. Note however that this change only delays the onset of the discrepancy for the $\mathrm{H} \alpha$ fit, so is introduced here mostly for aesthetic reasons. We find that lowering the density exponent down to 8 and even 6 leads to an increase in the $\mathrm{H} \alpha$ strength but also produces a very severe mismatch of the $\mathrm{Ca}$ II lines, which become both stronger and broader than observed. We find that indeed, maintaining a high density exponent of 10 leads to better fits to observations,
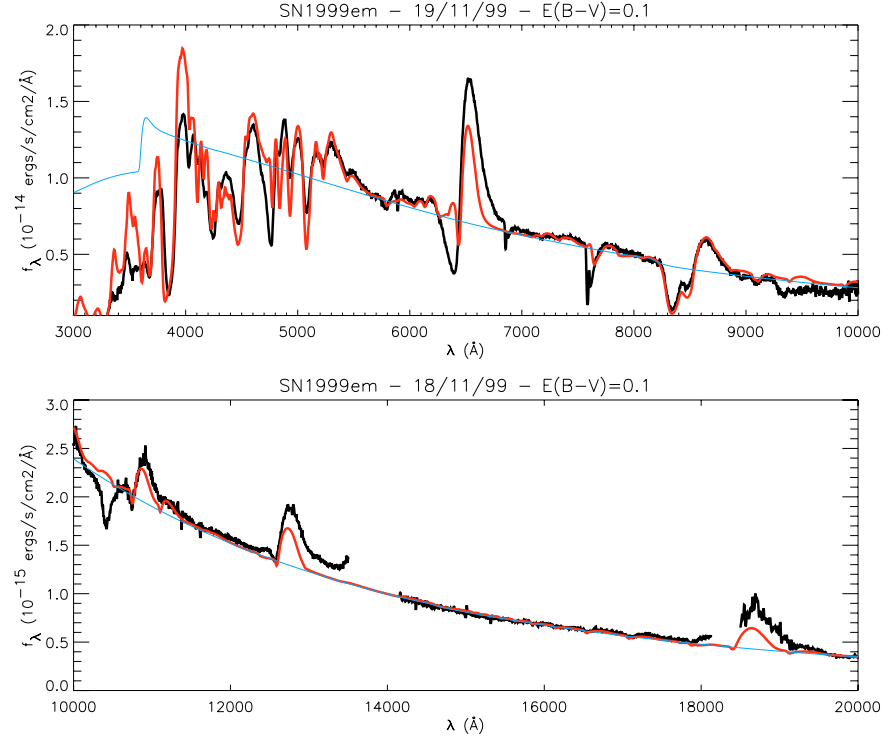

Fig. 7. Synthetic fit (red) to observations (black) taken on November 19th (H01; optical range, day 21) and 18th (H01; near-IR range). The near-IR model flux is scaled down by $15 \%$ compared to that in the optical, to allow the simultaneous match to both wavelength regions. The model properties are the following: $L_{*}=3.75 \times 10^{8} L_{\odot}$, $T_{\text {phot }}=6260 \mathrm{~K}, R_{\text {phot }}=12.9 \times 10^{14} \mathrm{~cm}, v_{\text {phot }}=5530 \mathrm{~km} \mathrm{~s}^{-1}, \rho_{\text {phot }}=$ $7.4 \times 10^{-14} \mathrm{~g} \mathrm{~cm}^{-3}$, and $n=10$. The model flux, which underestimates the observations by $10 \%$, is renormalized to the observed value at $6100 \AA$. (This figure is available in color in the electronic version.)

at least for the epochs covered here and our model assumptions (Paper I). Note also the presence of Fe II and Si II lines which fill in the $\mathrm{H} \alpha$ trough.

Omitting this difficulty of reproducing $\mathrm{H} \alpha$ and the Paschen series the quality of the fit is again satisfactory, with a good match to the strength and shapes of line features due to Fe II, Ti II, Ca II. The overall flux distribution is very well matched, with a slight overestimate of the flux in the blue. Another discrepancy is the slight overestimate of the $\mathrm{Ca}$ II/Fe II/Ti II blend around $3800 \AA$.

We observe a weak feature at $\sim 1.07 \mu \mathrm{m}$ which we attribute to the $\mathrm{C}$ I $(3 \mathrm{p}-3 \mathrm{~s})$ multiplet. However, to reproduce this weak feature, the carbon abundance had to be increased by a factor of 10 to $\mathrm{C} / \mathrm{He}=0.0017$, equivalent to a mass fraction of 0.0023. Similarly, a number of $\mathrm{CI}$ lines over the range 9060-9110 $\AA$ are seen, although a firm identification is established with the observation of the 5th of December 1999 (Sect. 3.2.8). Such a carbon abundance is compatible with chemical mixing prior to core-collapse, inducing a more moderate depletion of $\mathrm{C} / \mathrm{O}$ compared to $\mathrm{N}$. Accordingly, and following from the previous section, we identify the O I $7700 \AA$ line (adopting $\mathrm{O} / \mathrm{He}=10^{-2}$ ), as well as another oxygen feature in the near-IR at $1.13 \mu \mathrm{m}$, standing in the red wing of $\mathrm{P} \gamma$. Note that the latter also overlaps with a multiplets of $\mathrm{Mg}$ II (4p-3d) at $1.091-1.095 \mu \mathrm{m}$ (which represents about $30 \%$ of the $1.09 \mu \mathrm{m}$ feature).

An observed feature at $\sim 1.03 \mu \mathrm{m}$ is not predicted by CMFGEN, despite the numerous species included; this could 


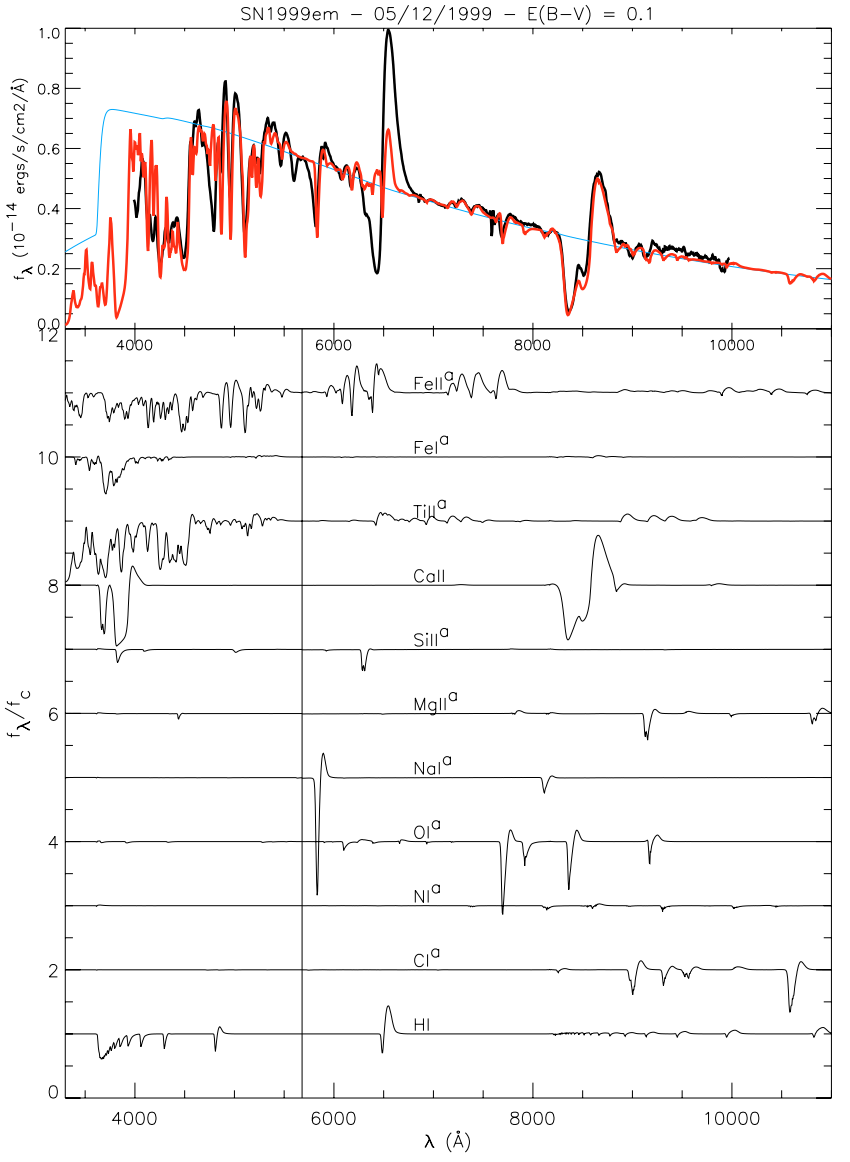

Fig. 8. Top: full (red) and continuum (blue) synthetic spectrum fits to observations (black) taken on the 5th of December 1999 (L02, day 37; this spectroscopic dataset is later used for distance determinations, in combination with the photometry on day 38 for which no spectra exist.) The model has the following parameters: $L_{*}=2 \times 10^{8} L_{\odot}$, $T_{\text {phot }}=5920 \mathrm{~K}, R_{\text {phot }}=10.9 \times 10^{14} \mathrm{~cm}, v_{\text {phot }}=3400 \mathrm{~km} \mathrm{~s}^{-1}, \rho_{\text {phot }}=$ $38.8 \times 10^{-14} \mathrm{~g} \mathrm{~cm}^{-3}$, and $n=10$. The model flux, which underestimates the observations by $10 \%$, is renormalized to the observed value at $7000 \AA$ A. Bottom: montage of rectified spectra computed by including bound-bound transitions of individual ionization species, ordered from the bottom by increasing atomic weight. For species labeled with an "a", flux departure from unity (continuum level) is magnified by a factor of four beyond $5680 \AA$. (This figure is available in color in the electronic version.)

come from singly-ionized species, or perhaps Sc or $\mathrm{Ba}$, not accounted for at present.

\subsubsection{Observation of the 5th of December 1999}

We present synthetic fits (color) to the observations (black) taken on the 5th of December 1999 (L02) in Fig. 8. The corresponding model has the following parameters: $L_{*}=2 \times 10^{8} L_{\odot}$, $T_{\text {phot }}=5920 \mathrm{~K}, R_{\text {phot }}=10.9 \times 10^{14} \mathrm{~cm}, v_{\text {phot }}=3400 \mathrm{~km} \mathrm{~s}^{-1}$, $\rho_{\text {phot }}=38.8 \times 10^{-14} \mathrm{~g} \mathrm{~cm}^{-3}$, and $n=10$. The model flux, which underestimates the observations by $10 \%$, is renormalized to the observed value at $7000 \AA$. Also, to ease the identification of observed spectral features, made more numerous due to the reduced outflow expansion velocity compared to previous dates, we show, in the lower panel, rectified spectra obtained when only bound-bound transitions of a given ionization species (see label) are included, ordered upwards with increasing atomic mass.

The overall fit quality is good. Discrepancies are noticeable for $\mathrm{H} \alpha$, whose strength in absorption and emission is underestimated; we are currently investigating possible extra energy sources that could preserve the large spatial extent of Balmer/Paschen line formation, imposed by the morphology of $\mathrm{H} \alpha$ at late times. Contrary to previous epochs, the nitrogen abundance on this date has to be reduced to maintain a good fit to the $8200 \AA$ feature, whose strength is explained almost entirely by the $\mathrm{NaI}$ line (see below). We thus adopt $\mathrm{N} / \mathrm{He}=$ $10^{-3}$ (by number). The $7700 \AA$ feature was at early-times associated with atmospheric extinction alone, although on the 14th and 19th of November, the absorption seemed to stretch further to the red, suggesting a contribution from the $\mathrm{O} I(3 \mathrm{p}-3 \mathrm{~s})$ triplet at $7775 \AA$. On the 5th of December 1999, following the reduced expansion velocity in the line formation region, one can unambiguously observe a P-Cygni line profile shape, blending with the atmospheric absorption only in the trough and blueward. As for earlier models, the reproduction of this line requires enhancing the oxygen abundance to $\mathrm{O} / \mathrm{He}=10^{-2}$ (by number). However, this leads to an overestimated strength for the O I (3s-3p) triplet line at $7984 \AA$; other O I lines at $8446 \AA$ and $9264 \AA$ overlap with Ca II, or Mg II and C I lines and are therefore not particularly useful as abundance constraints. We retain the same carbon abundance as in Sect. 3.2.7, where the near-IR C I feature at $1.07 \mu \mathrm{m}$ required an enhancement, with $\mathrm{C} / \mathrm{He}=1.7 \times 10^{-3}$. However, here, a clearly-defined $\mathrm{C}$ I feature is observed, stemming from the (3p-3s) multiplet covering the range $9060-9110 \AA$. We associate the $9200 \AA$ feature with the $\mathrm{Mg}$ II (4p-4s) doublet at 9218-9244 $\AA$, overlapping with the O I multiplet at $9260 \AA$ (note that we adopt a solar magnesium abundance). Finally, the absorption strength of the NaI(3p-3s) doublet at 5890-5895 $\AA$ is well matched with a factor of four enhancement compared to solar (Prantzos et al. 1986).

\subsection{Discussion of model results}

The models presented in the previous section provide satisfactory fits to our sample of spectrophotometric observations of SN1999em. We now investigate the overall accuracy of these model parameters, focusing on chemical abundances (Sect. 3.3.1), outflow ionization/temperature and reddening (Sect. 3.3.2), and photospheric velocity (Sect. 3.3.3).

\subsubsection{Chemical abundances}

Using spectroscopic observations during the photosphericphase evolution of type II SN, we anticipate inferring an outflow composition in conformity with $\mathrm{CNO}$-cycle equilibrium values, typical of the envelope of the corresponding massive star progenitor, with at most traces of elements produced through explosive nucleosynthesis. Indeed, adopting a blue- or red-supergiant surface composition (Prantzos et al. 1986) allows a good match to observed features of all species, for both their absolute and relative strengths. But let us now investigate 

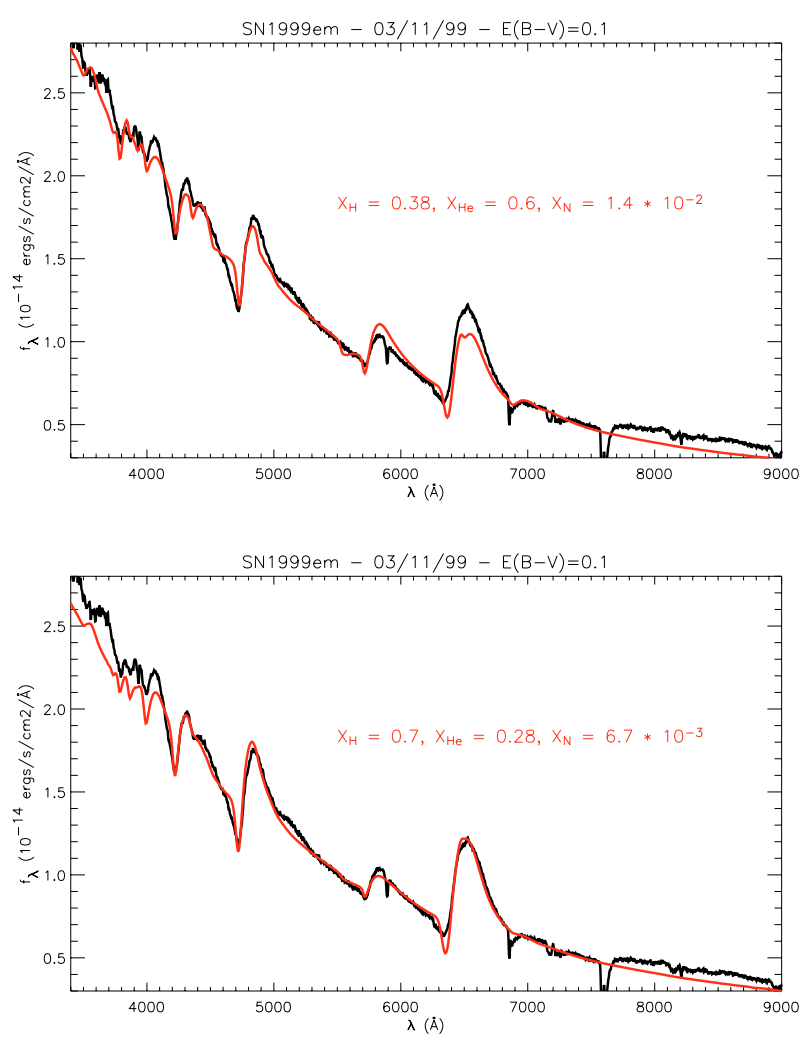

Fig. 9. Synthetic fits (red) to observations (black) of SN1999em on the 3rd of November 1999 (H01; day 5) using a model with the same parameters as those given in Sect. 3.2.3 (see also Fig. 3) but having a more (top) or less (bottom) evolved chemical composition than the baseline model described in Sect. 3.1. (This figure is available in color in the electronic version.)

how such line strengths are affected by a relative modulation of the outflow $\mathrm{H} / \mathrm{He} / \mathrm{CNO}$ chemistry, as would result from a distinct evolutionary stage or chemical mixing efficiency (linked to, e.g., rotation and/or magnetic fields).

We show in Fig. 9 a comparison between the observations of the 3rd of November 1999 (Sect. 3.2.3) and two synthetic spectra corresponding to more (top) or less (bottom) evolved progenitor stars. For the former, we adopt $X_{\mathrm{H}}=0.38, X_{\mathrm{He}}=$ $0.60, X_{\mathrm{C}}=3.1 \times 10^{-4}, X_{\mathrm{N}}=1.4 \times 10^{-2}$, and $X_{\mathrm{O}}=2.4 \times 10^{-4}$; for the latter, we use $X_{\mathrm{H}}=0.7, X_{\mathrm{He}}=0.28, X_{\mathrm{C}}=1.44 \times 10^{-4}$, $X_{\mathrm{N}}=6.7 \times 10^{-3}$, and $X_{\mathrm{O}}=1.13 \times 10^{-4}$.

The modulation in hydrogen abundance translates into a variation of the density of free-electrons in these fully ionized models: the higher the value of $X_{\mathrm{H}}$, the higher the electronscattering optical depth, either at the photosphere or at the model base. Here, we find that the photosphere for the $X_{\mathrm{H}}=0.7$ model is located $5 \%$ further out than the $X_{\mathrm{H}}=0.38$ model, at a cooler temperature $(10270 \mathrm{~K}$ rather than $11200 \mathrm{~K})$, explaining the slightly "cooler" SED for the hydrogen-richer model. Beside this slight change in the slope of the SED, the strength of individual features is noticeably altered.

In the $X_{\mathrm{H}}=0.38$ model, Balmer line strengths are significantly underestimated, and He I $5875 \AA$ overestimated. We also find that the increased nitrogen abundance leads to the overestimate of the strength of N II features around $5600 \AA$ and $4600 \AA$. A comparison of Figs. 9 with 3 shows that the depleted hydrogen model is clearly of inferior quality compared to our best fit model. With a solar $\mathrm{H} / \mathrm{He}$ ratio the changes relative to our best fit model are smaller, and it is more difficult to distinguish between the models. The sensitivity to changes in hydrogen, helium, and nitrogen composition is to be contrasted, for example, with Eastman \& Kirshner (1989), who required an unrealistically high helium abundance to reproduce the He I $5875 \AA$ line profile in the early spectra of SN1987A.

For carbon and oxygen, the situation is less clear: the outflow ionization is too low to lead to $\mathrm{C}$ II or O II lines anywhere in the UV, optical, and near-infrared. At a stage where hydrogen recombines in the outflow, the ionization is suitable for the C I line to appear around $9000 \AA$, whose fitting requires an enhancement of about a factor of ten above that of the baseline model (Sect. 3.2.8). Similarly, the same carbon abundance enhancement is needed to explain the feature at $1.07 \mu \mathrm{m}$, observed in the spectrum of the 19th of November (Fig. 7). We find a similar situation for O I lines, which appear in the red part of the spectrum only at late times, and require a significant enhancement compared to $\mathrm{CNO}$-cycle equilibrium values. Most notably, the O I lines at $7700 \AA$ and $1.13 \mu \mathrm{m}$ require to have $\mathrm{O} / \mathrm{He}$ in the vicinity of 0.01 (by number).

Overall, this investigation gives credence to our baseline model composition (Sect. 3.1) at early times, but with $\mathrm{C} / \mathrm{O}$ abundances enhancing, as times progresses, above $\mathrm{CNO}$-cycle equilibrium values; this suggests enhanced (rotational) mixing in the envelope of the progenitor star prior to collapse, or the appearance of the deeper and more chemically-evolved layers after just a few weeks past core-collapse.

\subsubsection{Outflow ionization, effective temperature, and reddening}

In the parameter space of photospheric-phase type II SN (Paper I), the similar effects produced by temperature and reddening modulations on the synthetic SED motivate a joint discussion.

In Fig. 10, we present spectroscopic observations of SN1999em taken on the 5th of November 1999 (Sect. 3.2.4 and Fig. 4), but now overplotted with the SED from models with decreasing photospheric temperatures: $T_{\text {phot }}=9560 \mathrm{~K}$ (blue), $T_{\text {phot }}=9200 \mathrm{~K}$ (red), and $T_{\text {phot }}=8850 \mathrm{~K}$ (green). Within the corresponding range of outflow ionization, the reduction of the UV flux is not so much caused by the decrease in $T_{\text {phot }}$, than by the opacity shift following the recombination from Fe III to Fe II in the SN outflow (as well as the opacity increase from numerous doubly-ionized metal species). In the optical, this shift has little bearing and the synthetic SED changes solely through the modest variation in $T_{\text {phot }}$.

Note, however, that applying a reddening modulation to the model corresponding to the red line in Fig. 11 leads to a similar modulation: the relatively-enhanced UV-extinction (Cardelli et al. 1988) echoes the strong line-blanketing due to metals discussed above. The relatively-reduced optical-extinction echoes the weak SED sensitivity to small $T_{\text {phot }}$ variations. Overall, a change in $E(B-V)$ of 0.05 or in $T_{\text {phot }}$ of $\sim \pm 500 \mathrm{~K}$ have comparable impacts on the SED in the present parameter space. 


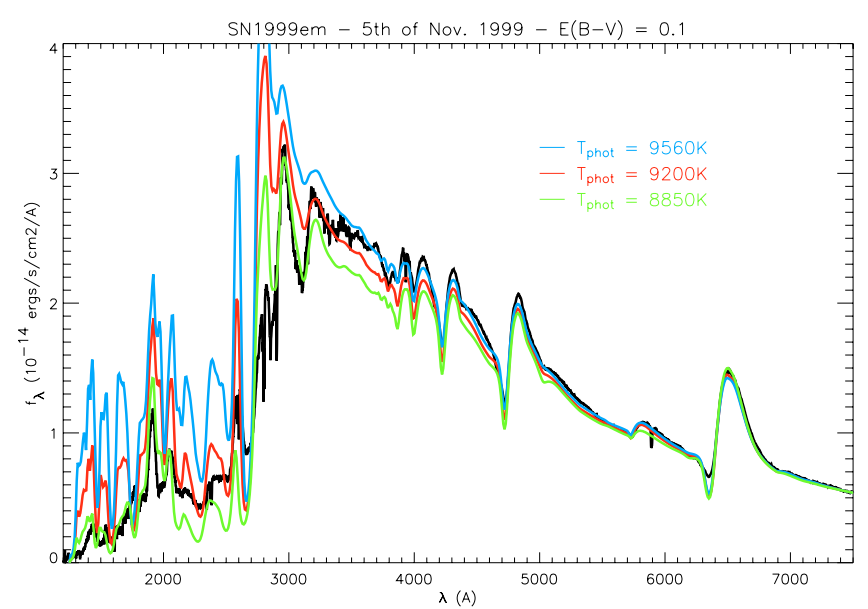

Fig. 10. Illustration of the impact of a temperature change on the synthetic SED, implemented through a $20 \%$ increase (blue) or decrease (green) in luminosity (at constant base radius) compared to the model presented in Fig. 4 of Sect. 3.2.4 (red) and observations (black). All other model parameters are held fixed. (This figure is available in color in the electronic version.)

Early-epoch multi-wavelength observations are thus very important: metal line-blanketing is then only moderate and helps disentangle between temperature and reddening effects; the UV range is also essential since this is where tests on the extinction magnitude are most sensitive.

\subsubsection{Photospheric velocity}

In Sect. 5 of Paper 2, we discussed how the velocity at maximum absorption in a sample of optical-range synthetic P-Cygni line profiles (i.e. Balmer lines and Fe II5169 $\AA$ ) relates to the corresponding model photospheric velocity. Contrary to the usually held belief, such a measurement on optically-thick lines does not systematically overestimate the model photospheric velocity. For any given ray, with impact parameter $p$, the location of maximum absorption for the flux-like quantity $p \cdot I(p)$ is exterior to the (continuum) photosphere, but for increasing $p$, this location shifts to smaller line-of-sight velocities, ultimately reaching line center. The total line flux is the integral of $p \cdot I(p)$ over all $p$, and thus reflects this range of velocities for the region of maximum-absorption. We find that although the velocity at maximum absorption for an optically-thick line always overestimates $v_{\text {phot }}$ for the ray with impact parameter $p=0$, it can either overestimate or underestimate $v_{\text {phot }}$ when the total line profile is considered. In practice, we identified few parameters that intervene in this correlation, the most important being the optical-depth of the line considered, which is related to the outflow ionization state, and the density gradient in the line formation region. These are physical entities that are difficult to constrain without detailed modeling.

Second, P-Cygni line profiles have shapes that make the location of maximum absorption difficult to identify. This problem is severe at early times (say for the first week after explosion), when the expansion velocity in the continuum- and line-forming regions is much larger and the flux in the lines departs little from that in the continuum, making line features

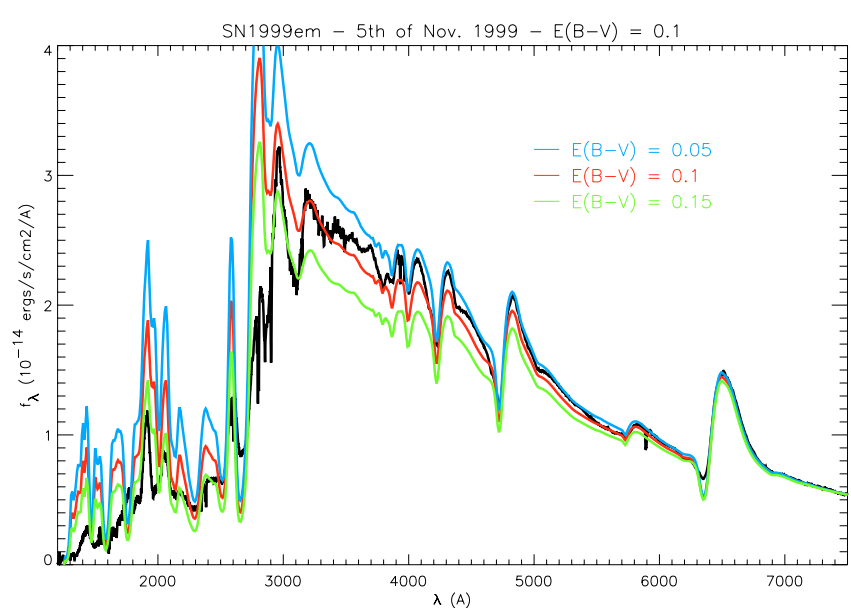

Fig. 11. Illustration of the effect of reddening modulations on the SED. We show the model and observations for the 5th of November 1999 (Fig. 4, day 7), reddening the synthetic SED with $E(B-V)$ of 0.05 (blue), 0.1 (red), and 0.15 (green). Note how, in this parameter range, a 0.05 change in $E(B-V)$ is comparable to a $\sim \pm 500 \mathrm{~K}$ shift in photospheric temperature (Fig. 10). (This figure is available in color in the electronic version.)

quite weak. This can be understood in the following way: suppose we take a model and increase its photospheric velocity: to first order, this will not change level populations, outflow ionization etc. and thus the total flux in all lines will be essentially the same, but the flux per-velocity-bin will be reduced, the more so for larger velocities. In practice, inspection of the first spectra for, e.g., SN1987A and SN1999em, show both very broad and very weak P-Cygni troughs, the region of maximum absorption covering few thousand $\mathrm{km} \mathrm{s}^{-1}$. In view of the argument discussed in the preceding paragraph, it is then difficult to infer a meaningful and accurate velocity for the photosphere.

Third, the assumption of a unique radius for the photosphere might not be appropriate on physical grounds. Multidimensional radiation-hydrodynamics simulations of corecollapse SN (Burrows et al. 1995; Janka \& Müller 1996) predict explosions with a systematic departure from sphericity, both from the large-scale (following, e.g., the core and envelope rotation) and small-scale (following, e.g., Rayleigh-Taylor instabilities) viewpoints. Such effects are expected to make the photosphere quite "fuzzy" at later times (say one month after explosion), showing a range of spatial scales for different lines of sight and a significant dispersion in the expansion velocity.

To illustrate the difficulty in assessing the photospheric velocity from P-Cygni line profiles, we show in Fig. 12 synthetic fits (red) to the observation (black) of SN1999em taken on the 9th of November 1999. The models shown have identical parameters to the one presented in Sect. 3.2.5 departing only from its photospheric velocity $v_{\text {phot }, 0}=7960 \mathrm{~km} \mathrm{~s}^{-1}$ : if the model photospheric velocity is $10 \%$ higher (top), the $\mathrm{H} \alpha$ width is overestimated but $\mathrm{H} \beta$, Ca II $3800 \AA$, and Fe II $5159 \AA$ are better fitted than in the model with a $10 \%$ slower photospheric velocity (bottom). But all three models fit almost equally well the observations so that there is a physically-meaningful uncertainty in photospheric velocity at the $10 \%$ level. While it is possible to measure precise velocities from the location of 

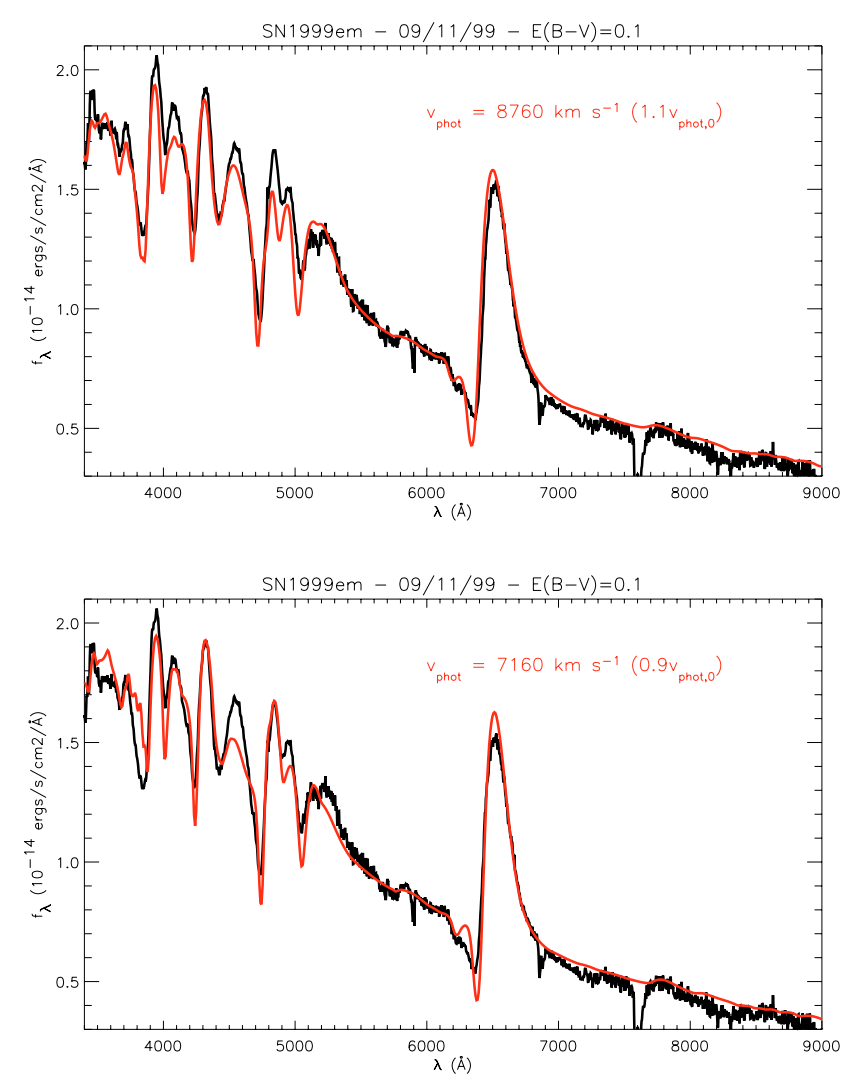

Fig. 12. Top: synthetic fits (red) to observations (black) of SN1999em on the 9th of November 1999 (H01; day 11) using a model with the same parameters as those given in Sect. 3.2.5 (see also Fig. 5) except for a photospheric velocity enhanced by $10 \%\left(v_{\text {phot }}=8760 \mathrm{~km} \mathrm{~s}^{-1}\right.$, $v_{\text {phot }, 0}=7960 \mathrm{~km} \mathrm{~s}^{-1}$ ). Bottom: same as above, but this time the photospheric velocity is reduced by $10 \%\left(v_{\text {phot }}=7160 \mathrm{~km} \mathrm{~s}^{-1}\right)$ compared to that of the reference model of Sect. 3.2.5. (This figure is available in color in the electronic version.)

maximum absorption in some P Cygni profiles, the errors associated with such measurements significantly underestimate the errors in the inferred photospheric velocity.

Thus, the inference of the photospheric velocity is made difficult by the ambiguity of interpreting line width and velocity at maximum absorption in P-Cygni profiles, as well as, perhaps, the physical velocity range spanned by the line formation region of different species.

For later use in distance determinations, we thus choose the model photospheric velocity obtained in Sect. 3.2 and given in Table 2; direct measurements on the observed spectra are more precise but the correspondence with $v_{\text {phot }}$ is unclear. For all dates, the overall quality of our fits is good, which gives us confidence that our model photospheric velocity is a meaningful reflection of the expansion velocity of the photosphere, say to within $\pm 10 \%$ at most.

\section{The distance to SN1999em}

Let us now determine the distance $D$ to $\mathrm{SN} 1999 \mathrm{em}$ - to do so we will use 5 distinct, but closely related, approaches. For the first 2 approaches we use the "standard" form of the EPM (H01, L02), in combination with either E96/H01's or Paper II's correction factors. In the third approach we use the models to derive the temperatures which are then combined with Papers II's correction factors. With the final EPM approach, we use both temperatures and correction factors inferred from the best fit models. Finally, we use the SEAM approach of Baron et al. (2004).

As discussed in Sects. 2-3, we selected eight epochs (October 30th, November 1st, 3rd, 5th, 9th, 14th, 19th, and December 5th) for which CMFGEN fitted well both continuum and line fluxes. Relative to the discovery date HJD 2451 480.94, these correspond to days 1, 3, 5, 7, 11, 16, 21 , and 38. No spectra were taken on day 38: for that date, given the very slow spectroscopic and photometric evolution at such late times, we combine the spectrum on day 37 with the photometry of day 38 . Whatever the approach, we use the relevant model photospheric velocity. Note also that, in this section, all synthetic magnitudes are computed using the transmission functions and zero points of $\mathrm{H} 01$.

\subsection{Inference with the EPM}

A key component of the EPM (E96, H01, L02, Paper II) lies in the correspondence between correction factors and blackbody color temperatures $T_{\mathrm{c}}$, defined from detailed models of type II SN spectra (E96; Paper II). In Paper II, we showed that our correction factors possess similar properties to those found in E96, albeit offset upwards by $\sim 20 \%$. At a given $T_{\mathrm{c}}$, correction factors show an intrinsic scatter of $\sim 10-20 \%$, roughly independent of $T_{\mathrm{c}}$. However, if caused by uncertainties in $T_{\mathrm{c}}$, this scatter increases dramatically at low- $T_{\mathrm{c}}$. Tabulated correction factors may also be inadequate to account for the blackbody assumption, if changes in magnitudes are due to variations in expansion velocity or metallicity, rather than changes, e.g., in temperature or the strength of flux dilution. Hence, we anticipate that using tailored models for each individual date will be more consistent and thereby improve the use of the EPM technique for distance determinations.

In this section, we show a sequence of computations of distances based on distinct sets of quantities $\theta_{S}, \xi_{S}$ and $T_{S}$. Here, $S$ refers to one of the three bandpass combinations, $\{B, V\}$, $\{B, V, I\}$, and $\{V, I\}, \theta_{S}$ to the quantity $R_{\text {phot }} / D$ (not known individually), $\xi_{S}$ to correction factors, and $T_{S}$ to the blackbody color temperature, each of the last three quantities corresponding to a selected bandpass combination $S$. An error analysis of the EPM technique and a description of the technique used to derive the distances are given in Appendices A and B.

In Table 3, we show such a set computed with the "standard" form of the EPM (L02, H01) and using H01's correction factors. Restricting first the observational dataset to the first seven dates (prior to and excluding the 5th of December $1999)^{2}$, we obtain increasing distances for the three bandpass combinations, $\{B, V\},\{B, V, I\}$, and $\{V, I\}$, from $8.6 \mathrm{Mpc}$,

2 Throughout the paper we use observational data sets containing either 7 or 8 dates. This serves two purposes: first, $\mathrm{H} \alpha$ in the last data set is not well fitted, meaning that systematic errors could, potentially, be large. Second, the effect of the longer time baseline, which helps to increase the accuracy of the explosion date, can be discerned. 
Table 3. Table of EPM quantities $\theta_{S}\left(10^{8} \mathrm{~km} \mathrm{Mpc}^{-1}\right), \xi_{S}$, and $T_{S}(\mathrm{~K})$, for the eight epochs covered by our analysis (i.e. from the 30 th of October till the 5th of December 1999; see Sect. 2). Also given are the resulting distance and explosion dates inferred. Days and dates are relative to JD 2451480.94 .

\begin{tabular}{|c|c|c|c|c|c|c|c|c|c|}
\hline \multicolumn{10}{|c|}{ EPM combined with minimization technique and $\xi$ prescription from $\mathrm{H} 01$} \\
\hline \multirow[t]{2}{*}{ Day } & \multicolumn{3}{|c|}{$\begin{array}{c}\text { Angular size } \\
\left(10^{8} \mathrm{~km} \mathrm{Mpc}^{-1}\right)\end{array}$} & \multicolumn{3}{|c|}{ Correction factor } & \multicolumn{3}{|c|}{$\begin{array}{c}\text { Temperature } \\
(\mathrm{K})\end{array}$} \\
\hline & $\theta_{B V}$ & $\theta_{B V I}$ & $\theta_{V I}$ & $\xi_{B V}$ & $\xi_{B V I}$ & $\xi_{V I}$ & $T_{B V}$ & $T_{B V I}$ & $T_{V I}$ \\
\hline 1.0 & 6.28 & 7.78 & 8.84 & 0.405 & 0.425 & 0.440 & 16860 & 13513 & 11543 \\
\hline 3.0 & 6.73 & 8.31 & 9.37 & 0.401 & 0.423 & 0.440 & 16476 & 13241 & 11383 \\
\hline 5.0 & 8.16 & 9.14 & 9.82 & 0.382 & 0.416 & 0.438 & 14394 & 12312 & 10998 \\
\hline 7.0 & 8.46 & 9.89 & 10.87 & 0.377 & 0.413 & 0.436 & 13801 & 11479 & 10070 \\
\hline 11.0 & 11.10 & 11.48 & 11.85 & 0.367 & 0.412 & 0.436 & 11271 & 10214 & 9477 \\
\hline 16.0 & 15.77 & 13.89 & 12.23 & 0.461 & 0.434 & 0.437 & 7747 & 8532 & 9365 \\
\hline 21.0 & 16.45 & 15.47 & 13.89 & 0.600 & 0.478 & 0.445 & 6514 & 7411 & 8404 \\
\hline \multirow[t]{2}{*}{38.0} & 17.51 & 17.58 & 16.98 & 1.202 & 0.652 & 0.482 & 4688 & 5777 & 7043 \\
\hline & \multicolumn{3}{|c|}{$B V$ set } & \multicolumn{3}{|c|}{$B V I$ set } & \multicolumn{3}{|c|}{$V I$ set } \\
\hline & \multicolumn{9}{|c|}{ Using the first 7 dates only } \\
\hline$D$ & \multicolumn{3}{|c|}{$8.6 \pm 0.8$} & \multicolumn{3}{|c|}{$9.7 \pm 1.0$} & \multicolumn{3}{|c|}{$11.7 \pm 1.5$} \\
\hline$t_{\exp }$ & \multicolumn{3}{|c|}{$-4.4 \pm 0.9$} & \multicolumn{3}{|c|}{$-7.0 \pm 1.4$} & \multicolumn{3}{|c|}{$-10.4 \pm 2.1$} \\
\hline & \multicolumn{6}{|c|}{ Using 8 dates } & \\
\hline$D$ & \multirow{2}{*}{\multicolumn{3}{|c|}{$\begin{array}{c}8.0 \pm 0.6 \\
-3.9 \pm 0.7\end{array}$}} & \multicolumn{3}{|c|}{$8.8 \pm 0.7$} & \multicolumn{3}{|c|}{$10.1 \pm 0.9$} \\
\hline$t_{\exp }$ & & & & \multicolumn{3}{|c|}{$-5.9 \pm 1.0$} & \multicolumn{3}{|c|}{$-8.4 \pm 1.4$} \\
\hline
\end{tabular}

Table 4. Same as Table 3, this time using Paper II's $\xi$-prescription.

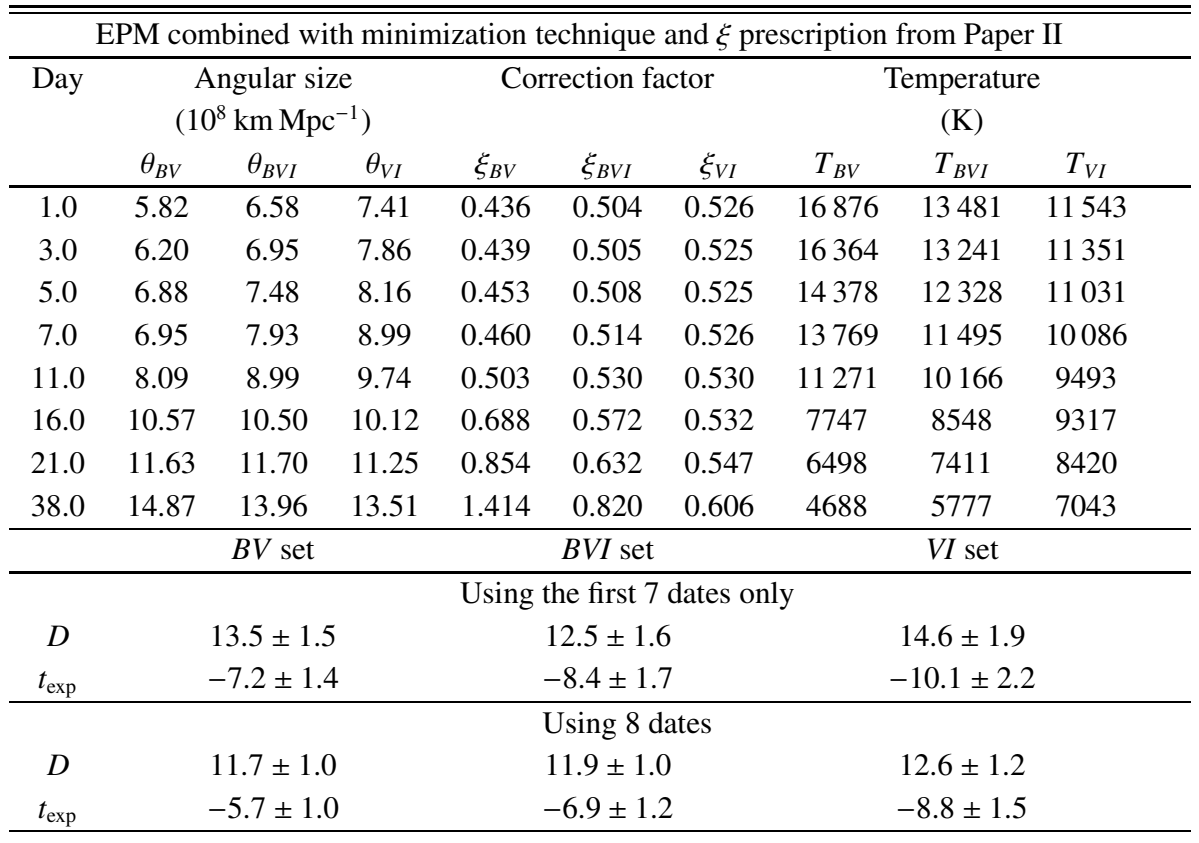

to $9.7 \mathrm{Mpc}$, and $11.7 \mathrm{Mpc}$, while the explosion date $t_{\mathrm{exp}}$ moves back in time in the same order, from -4.4 days, to -7.0 days, and -10.4 days. The disparity amongst bandpass combinations is large, especially for the explosion dates, and unsatisfactory since the EPM should yield similar distances and explosion times whatever choice of bandpass combination, provided correction factors across the range of color temperatures are appropriate. Adding the eighth date to the dataset leads to systematically smaller distances which are in better agreement but the explosion dates still differ substantially. The derived distance is approximately $8.7 \mathrm{Mpc}$ which is $75 \%$ of the Cepheid-distance to NGC 1637 and SN1999em.

We show in Table 4 the corresponding quantities obtained with Paper II's $\xi$-prescription. These new correction factors are indeed always higher than those shown in Table 3 for the same date, but the magnitude of the difference varies from date to date. Hence, despite the preserved blackbody color temperatures between Tables 3 and 4, the non-uniform offset in correction factors between the two prescriptions induces a non-uniform offset in individual $\theta_{S}$. Resulting distances are 
Table 5. Same as Table 3, this time using blackbody color temperatures from Sect. 3, and Paper II's $\xi$-prescription.

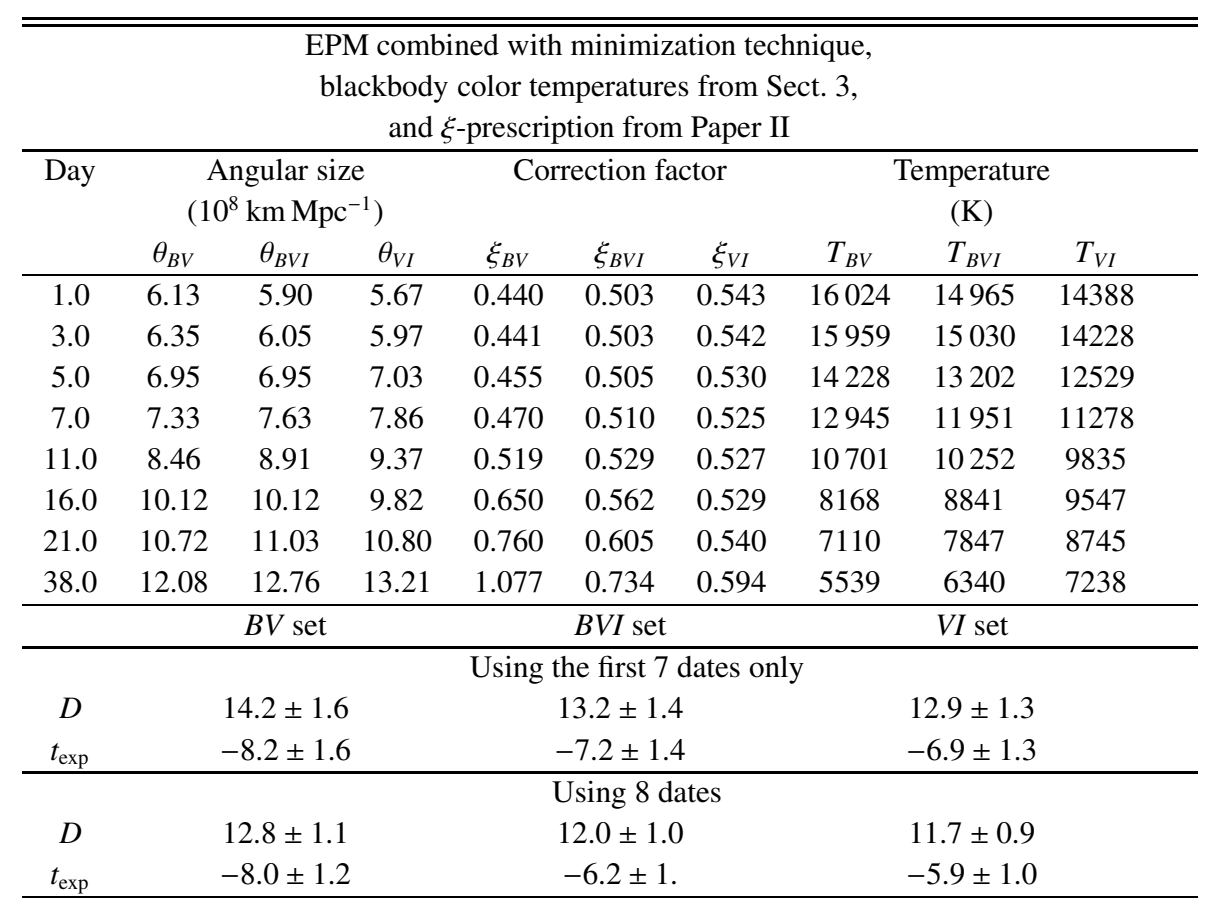

systematically larger, using either 7 or 8 dates, and the dispersion in the explosion times is reduced.

We have investigated whether enforcing a fixed scaling $x>1$ on correction factors given by H01 would clarify these behaviors. As above, we find it introduces no noticeable modulation of the inferred color temperature (these remain constant at the one percent level). However, the individual values $\theta_{S}$ obtained are then scaled by $1 / x$, basically leaving $\xi_{S} \times \theta_{S}$ constant. The resulting distances in that case are also scaled by $x$, but the explosion dates are now essentially unchanged. Using H01 or Paper II correction factors thus modifies both the inferred time of explosion and the distance, an effect arising from the nonuniform scaling in correction factors between $\mathrm{H} 01$ and Paper II; in other words, the differential variation of $\xi_{S}$ with $T_{S}$ affects distinct dates differentially.

An important factor influencing the accuracy of the distance determinations is the incompatibility, at a given date, between color temperatures $T_{S}$ obtained here (Tables 3 and 4), and those previously computed on corresponding CMFGEN model SEDs (Table 2 in Sect. 3.2). For bandpass combinations $\{B, V, I\}$ and $\{V, I\}$, model values of $T_{S}$ are systematically larger than those deduced from the minimization technique performed on observations, while for $\{B, V\}$, they are smaller at high and larger at low $T_{B V}$. This color-mismatch is likely to stem from the flux offset between synthetic and observed spectra, leading to an over- or under-estimate of the corresponding bandpass magnitudes on which the computation of colortemperatures is based. Figure 3 shows such a situation where the observed $I$ band flux is substantially underestimated by our model - note that the flux calibration may also be at fault, especially at longer optical wavelengths where atmospheric extinction operates. To be more quantitative, we provide an estimate of the effect of flux errors on the derived angular diameter in Appendix A (Table A.1).

We now compute the EPM-distance using the model colortemperatures. First, we use the $\xi$-prescription from Paper II and show resulting EPM quantities in Table 5. Distances derived using the first 7 dates are systematically higher than the Cepheid distance, but have large errors. The explosion dates, within errors, are consistent. With 8 dates, all distances agree within 1 sigma of the Cepheid distance of $11.7 \mathrm{Mpc}$ (L03) and the SEAM distance of 12.4 Mpc (B04, see next section). Because the observational and model color temperatures show filter set and epoch dependent differences, the changes in the derived distances between those shown in Tables 4 and 5 are not systematic or uniform.

Finally, combining both color-temperatures and correction factors from the models of Sect. 3.2, we find even better agreement in the distance predicted between the different band passes, of $11.7 \mathrm{Mpc}, 11.6 \mathrm{Mpc}$, and $11.5 \mathrm{Mpc}$, in the usual sequence (Table 6). This is expected since the same models are used - indeed the differences, typically less than $2 \%$, must simply reflect errors in interpolations etc arising in the application of the EPM technique. The corresponding explosion dates are -6.2 days, for all three data sets. In Fig. 13, we show a linear fit of the form $\theta / v_{\text {phot }}=\left(t-t_{\exp }\right) / D$ to the BV data shown in Table 6.

\subsection{Inference based on CMFGEN synthetic SEDs}

The Spectral-fitting Expanding Atmosphere Method (SEAM; B04; Mitchell et al. 2002) is an attractive alternative to the EPM: each observed SED is directly fitted with a synthetic SED, produced by a detailed model atmosphere calculation for the corresponding date, avoiding necessary corrections for 
Table 6. Same as Table 3, this time using blackbody color temperatures and $\xi$-values from Sect. 3 .

\begin{tabular}{|c|c|c|c|c|c|c|c|c|c|}
\hline \multicolumn{10}{|c|}{$\begin{array}{l}\text { EPM combined with blackbody color temperatures } \\
\text { and } \xi \text {-values from Sect. } 3\end{array}$} \\
\hline \multirow[t]{2}{*}{ Date } & \multicolumn{3}{|c|}{$\begin{array}{c}\text { Angular size } \\
\left(10^{8} \mathrm{~km} \mathrm{Mpc}^{-1}\right)\end{array}$} & \multicolumn{3}{|c|}{ Correction factor } & \multicolumn{3}{|c|}{$\begin{array}{c}\text { Temperature } \\
(\mathrm{K})\end{array}$} \\
\hline & $\theta_{B V}$ & $\theta_{B V I}$ & $\theta_{V I}$ & $\xi_{B V}$ & $\xi_{B V I}$ & $\xi_{V I}$ & $T_{B V}$ & $T_{B V I}$ & $T_{V I}$ \\
\hline 1.0 & 5.82 & 5.90 & 5.97 & 0.462 & 0.500 & 0.519 & 16024 & 14965 & 14388 \\
\hline 3.0 & 6.50 & 6.58 & 6.65 & 0.430 & 0.462 & 0.488 & 15959 & 15030 & 14228 \\
\hline 5.0 & 7.11 & 7.18 & 7.26 & 0.443 & 0.488 & 0.513 & 14228 & 13202 & 12529 \\
\hline 7.0 & 7.78 & 7.86 & 7.86 & 0.443 & 0.494 & 0.526 & 12945 & 11951 & 11278 \\
\hline 11.0 & 9.37 & 9.44 & 9.37 & 0.469 & 0.500 & 0.526 & 10701 & 10252 & 9835 \\
\hline 16.0 & 10.20 & 10.20 & 10.27 & 0.646 & 0.558 & 0.507 & 8168 & 8841 & 9547 \\
\hline 21.0 & 11.85 & 12.01 & 12.16 & 0.685 & 0.558 & 0.481 & 7110 & 7847 & 8745 \\
\hline \multirow[t]{2}{*}{38.0} & 12.16 & 12.38 & 12.83 & 1.072 & 0.755 & 0.608 & 5539 & 6340 & 7238 \\
\hline & \multicolumn{3}{|c|}{$B V$ set } & \multicolumn{3}{|c|}{$B V I$ set } & \multicolumn{3}{|c|}{$V I$ set } \\
\hline & \multicolumn{9}{|c|}{ Using the first 7 dates only } \\
\hline$D$ & \multicolumn{3}{|c|}{$12.4 \pm 1.2$} & \multicolumn{3}{|c|}{$12.4 \pm 1.3$} & \multicolumn{3}{|c|}{$12.4 \pm 1.3$} \\
\hline$t_{\mathrm{exp}}$ & \multicolumn{3}{|c|}{$-6.8 \pm 1.3$} & \multicolumn{3}{|c|}{$-6.9 \pm 1$} & \multicolumn{3}{|c|}{$-7.0 \pm 1.3$} \\
\hline & \multicolumn{9}{|c|}{ Using 8 dates } \\
\hline$D$ & \multicolumn{3}{|c|}{$11.7 \pm 1.0$} & \multicolumn{3}{|c|}{$11.6 \pm 1.0$} & \multicolumn{3}{|c|}{$11.5 \pm 0.9$} \\
\hline$t_{\exp }$ & \multicolumn{3}{|c|}{$-6.2 \pm 1.0$} & \multicolumn{3}{|c|}{$-6.2 \pm 1.0$} & \multicolumn{3}{|c|}{$-6.2 \pm 1.0$} \\
\hline
\end{tabular}

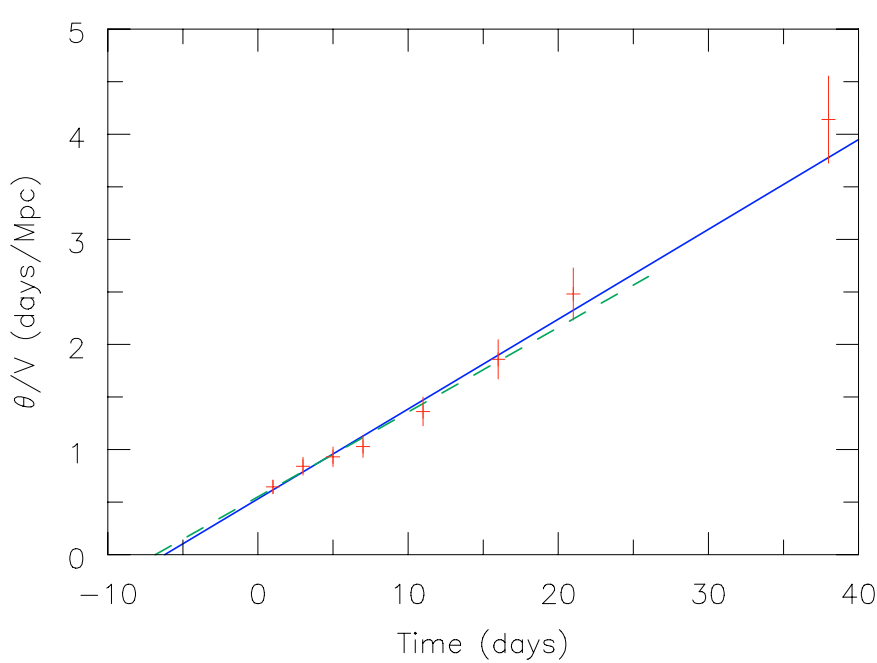

Fig. 13. Linear fit to the BV data shown in Table 6 for 8 data points (solid blue line) and 7 data points (broken green line). The reciprocal of the slope is the distance to the SN. The time is measured with respect to JD 2451480.9 . The vertical bars indicate $\pm 10 \%$. (This figure is available in color in the electronic version.)

approximating observed colors with those of a singletemperature blackbody (EPM; see previous section).

Nonetheless, the SEAM follows a similar procedure to the EPM. Indeed we would expect to derive very similar answers to that found using the EPM technique with detailed SN fitting. We first select a number of epochs for which photometric and spectroscopic observations are available and accurate, and for which we possess high quality fits to the observed SED. We then select a range of explosion times, and compute the photospheric radius expected for each date of observation, given by $R_{\text {phot }}(t)=v_{\text {phot }}(t)\left(t-t_{\text {exp }}\right)$, where $t$ is the observation date, $t_{\exp }$ the explosion date, and $v_{\text {phot }}(t)$ represents the corresponding photospheric velocity (the initial radius of each considered mass shell is neglected). As in the EPM, the assumption of homologous expansion, warranted past $\approx 1$ day after core-bounce, is essential.

In our approach, we do not evolve an original hydrodynamical input for the exploding envelope; we use instead an analytical description of the density distribution, and adjust the luminosity to match typical values expected from explosion models and former spectroscopic studies (for SN1999em, we use the Cepheid-distance to ensure our adopted luminosities are compatible with observed fluxes and magnitudes). For a chosen $t_{\exp }$ corresponds an $R_{\text {phot }}(t)$, usually different from that of the fitting model for that date; we thus scale the model luminosity in proportion with the square of the change in $R_{\text {phot }}(t)$, and update synthetic magnitudes accordingly. Then, for each choice of the explosion date $t_{\exp }$ and for each observation epoch $t$, we compute the distance modulus $\mu_{S}\left(t_{\exp }, t\right)$ for all bandpass sets $S=\{B, V\},\{B, V, I\}$, and $\{V, I\}$, given by $\mu_{S}=\left\langle\mu_{X}\right\rangle_{S}$, where $\mu_{X}=m_{X}-M_{X}-A_{X} ; X$ represents one of the band passes in the set $S ; m_{X}, M_{X}$, and $A_{X}$ are the apparent, absolute, and extinction magnitudes in bandpass $X$. Thus, for each chosen explosion date, we compute, for each set $S$, a distance modulus $\mu_{S}\left(t_{\exp }, t\right)$ averaged over all observation epochs. The distance modulus selected at the end corresponds to the $t_{\exp }$ that minimizes the scatter of $\mu_{S}\left(t_{\text {exp }}, t\right)$ over all epochs.

We show results for this sample of observations in Table 7. The results are grouped by triads. Using the adopted reddening $E(B-V)=0.1$ and only seven (eight) observations, we obtain a distance of $\sim 12.15 \mathrm{Mpc}(11.5 \mathrm{Mpc})$, in good agreement with the SEAM-distance of B04 $(12.4 \pm 2.5 \mathrm{Mpc})$ or the Cepheid distance of Leonard et al. (2003; $11.7 \pm 1 \mathrm{Mpc})$. Limiting the sample to the first four epochs only leads to a significant increase in the distance, to $\sim 15.2 \mathrm{Mpc}$ : the much reduced time-span makes this prediction unreliable. Note also that the 
Table 7. SEAM results for the explosion date and distance to SN1999em, under a variety of choices that highlight uncertainties: bandpass set, number of observation dates, reddening, and photospheric velocity values. Time is relative to JD 2451480.54 .

\begin{tabular}{ccccccc}
\hline \hline $\begin{array}{c}t_{\text {exp }} \\
\text { (days) }\end{array}$ & $\mu$ & $\begin{array}{c}d \\
(\mathrm{Mpc})\end{array}$ & $E_{B-V}$ & $\begin{array}{c}v_{\text {scale }} \\
\left(v_{\text {phot }}\right)\end{array}$ & $S$ & $n_{\text {obs }}$ \\
\hline-6.7 & 30.44 & 12.22 & 0.10 & 1.0 & $B V$ & 7 \\
-6.9 & 30.44 & 12.26 & 0.10 & 1.0 & $B V I$ & 7 \\
-6.8 & 30.41 & 12.10 & 0.10 & 1.0 & $V I$ & 7 \\
\hline-9.5 & 30.94 & 15.45 & 0.10 & 1.0 & $B V I Z$ & 4 \\
-9.1 & 30.90 & 15.14 & 0.10 & 1.0 & $V Z$ & 4 \\
-9.3 & 30.91 & 15.20 & 0.10 & 1.0 & $B V I$ & 4 \\
\hline-6.9 & 30.44 & 12.26 & 0.10 & 1.0 & $B V I$ & 7 \\
-6.8 & 30.58 & 13.05 & 0.05 & 1.0 & $B V I$ & 7 \\
-6.8 & 30.29 & 11.41 & 0.15 & 1.0 & $B V I$ & 7 \\
\hline-6.9 & 30.44 & 12.26 & 0.10 & 1.0 & $B V I$ & 7 \\
-7.0 & 30.66 & 13.57 & 0.10 & 1.1 & $B V I$ & 7 \\
-6.9 & 30.21 & 11.02 & 0.10 & 0.9 & $B V I$ & 7 \\
\hline-6.3 & 30.34 & 11.68 & 0.10 & 1.0 & $B V$ & 8 \\
-6.2 & 30.30 & 11.51 & 0.10 & 1.0 & $B V I$ & 8 \\
-6.0 & 30.25 & 11.22 & 0.10 & 1.0 & $V I$ & 8 \\
\hline
\end{tabular}

use of the $Z$-band has only a modest impact on the resulting distance.

If we assume that our adopted models, which fit well the observations with the choice of $E(B-V)=0.1$, would still fit the observations adequately with a modulation of the reddening by \pm 0.05 about this nominal value (see the discussion in Sect. 3.3.2), then such a reddening uncertainty introduces a distance uncertainty of $\approx \pm 0.8 \mathrm{Mpc}$ (equivalent to a \pm 0.15 range in distance modulus). However, this represents an upper limit since new models should be computed that fit better the observations with the newly adopted reddenings. When this is done, the change in derived temperatures and radii partially compensate for the change in reddening (Appendix A).

We find that a $\pm 10 \%$ error in $v_{\text {phot }}$ has a negligible impact on the inferred time of explosion (constrained by the relative evolution of magnitudes between different epochs) but affects noticeably the inferred distance: at a given epoch, smaller (larger) photospheric velocities mean smaller (larger) photospheric radii and thus smaller (larger) distances to match the observed magnitudes. Thus, by itself, the error on $v_{\text {phot }}$ (Sect. 3.3.3) introduces an uncertainty of $\pm 1 \mathrm{Mpc}$ in the inferred distance. Finally, we have added an eighth observation to our sample used so far (that of the 5th of December 1999). The average distance for the three bands used is then reduced by $\sim 0.5-1 \mathrm{Mpc}$ compared to that of the reference case.

\section{Conclusion}

In this paper, we have used the model atmosphere code CMFGEN (Papers I and II) to perform a quantitative spectroscopic analysis of the photospheric-phase evolution of SN1999em, a type II supernovae.

We obtain high-quality fits to flux-calibrated data for eight epochs (from the 30th of October until the 5th of December 1999), modeling the evolution of the optically-thick outflow from full-ionization to recombination. Indeed, we find that the spectroscopic changes are primarily due to the temperature decrease following expansion and radiation-leakage, associated with a decrease in the outflow ionization. The critical shift from Fe III to Fe II at $T_{\text {phot }} \sim 8000 \mathrm{~K}$, concomitant with the onset of hydrogen recombination, causes a dramatic increase in opacity, first in the UV and later in the optical, that alters considerably the overall shape of the spectral energy distribution. For fully or partially ionized models, we were able to reproduce, with good accuracy, the shape of both the spectral energy distribution and line profiles. The thus-constrained chemical composition is compatible with CNO-cycle equilibrium abundance values for the first month. At early times, the carbon and oxygen abundances are poorly constrained due to the lack of lines of CII and OII in the optical and near-IR. At later times, with further cooling of the outflow, $\mathrm{C}$ I and $\mathrm{O}$ I lines are seen in the 7000-12000 $\AA$ regions, requiring significant enhancements over the CNO-cycle equilibrium values; it is not clear if this corresponds to a genuine increase in the corresponding abundances as the photosphere recedes into deeper layers, or if this abundance pattern was identical at all previous times.

Significantly, there was no observational incentive to vary the metal composition, whatever the epoch, so we adopted a unique mixture corresponding to solar. In other words, over a 38-day period, we see no spectroscopic evidence of products from explosive-nucleosynthesis. Additionally, we find no evidence for a flatter density distribution at later times: for all epochs, we adopt a density exponent $n=10-12$ (Papers I, II) which permits satisfactory fits to line profile shapes. Finally, as for other groups (e.g. Mitchell et al. 2002), we have difficulty in reproducing Balmer (and Paschen, when available) line profiles, both in strength and width. This discrepancy might stem from a missing outflow energy source, perhaps, e.g., in the form of energy deposition from radioactive decay of ${ }^{56} \mathrm{Ni}$ (Mitchell et al. 2002), or result from the neglect of time-dependence effects (see, e.g., Utrobin \& Chugai 2005) in standard radiative transfer modeling of photospheric phase type II SN. We are currently studying this issue.

We also estimate the distance to SN1999em using a variety of approaches and compare with the reference value of Leonard et al. (2003) using NGC 1637 Cepheids. Although we reproduce the under-estimated EPM distance of Leonard et al. (2002) or Hamuy et al. (2001), there are non-trivial differences between our approach and assumptions, and these two works. First, our adopted photospheric velocities are higher or lower than theirs, depending on the epoch: systematically higher photospheric velocities lead to faster increasing photospheres, which under the same conditions of magnitude etc., require a higher distance - the difficulty here is that the difference in velocity is positive or negative at different dates. Second, the latest epoch is limited to day 21 ( 7 th observation in our sample) or day 38 (8th observation) after discovery - add $\sim 6.2$ days to time of core-collapse - in our approach rather than $\gtrsim 70$ days: there is a sensible incentive in lengthening the time baseline to reduce the errors (see Appendix), but as argued in Paper II, the corrupting effect of lines in all optical band passes as well as the likely energy contribution from radioactive decay make such late time observations less adequate for the EPM. Third, to extract the distance and time of explosion from our collection 
of $\{t, \theta / v\}$ data points, we use time as the independent variable, rather than $\theta / v$ : this seems a better approach since time is accurately known. Moreover, in our least-square fitting, we adopt constant fractional errors in $\theta / v$ rather than constant weights. We find that this approach yields distances that are systematically and at least $10 \%$ greater that those obtained by assuming $\theta / v$ as the independent variable and constant weights.

Applying the correction-factor recipe of Paper II leads to a higher distance, in good agreement with the Cepheid estimate. It thus seems that correction factors are indeed a major uncertainty in the EPM, the systematically lower values used previously playing a central role in the discrepancy (see, e.g., Baron et al. 2004). Overall, however, approaches based on tabulated correction factors lead to a significant disparity in distances obtained from different bandpass sets, in conflict with the nearconstancy, at early times, of the angular size of the photosphere with optical-wavelength. The problem likely stems from the intrinsic scatter of correction factors at a given color-temperature, obtained from observations alone or even adopted from tailored models.

When both color-temperatures and correction factors are adopted from individual best-fit models to each observation, the EPM technique delivers more consistent distance estimates between band passes. Slight differences persist from small but systematic discrepancies between the model and observed spectral distributions. But with such an approach, we obtain an EPM-distance of $11.5 \pm 1 \mathrm{Mpc}$, in agreement with the Cepheid estimate. Hence, while there is nothing fundamentally wrong with the EPM, the need for detailed model atmosphere calculations defeats the original purpose of the method.

We obtain another distance estimate of $12.2 \pm 2 \mathrm{Mpc}$ using synthetic spectra and magnitudes, minimizing the scatter in individual distance moduli for a range of adopted explosion dates. This approach is similar to the approach of Baron et al. (2004) and delivers a similar distance. The inferred explosion date is the 23rd of October 1999 with an uncertainty of 1 day this is approximately 4 days earlier than the estimate of Hamuy et al. (2001) but similar to that of Leonard et al. (2002) and Baron et al. (2004).

This study shows that there are a variety of reliable ways to determine distances with early-time photospheric-phase type II SN, with good prospects for cosmology. In forthcoming studies, we will reproduce the present analysis with a larger sample of objects, covering a wider range of distances or redshifts, and thereby attempt to provide an alternative, to using type Ia SN, for the cosmological distance scale.

Acknowledgements. We wish to thank Mario Hamuy for providing some of the spectra used in this study, as well as assistance with the photometry. We also thank Eddie Baron for providing the HST data for SN1999em taken on the 5th of November, as well as Doug Leonard for comments on a draft of this paper. D.J.H. gratefully acknowledges partial support for this work from NASA-LTSA grant NAG5-8211. Improved data for C I was kindly supplied by Bob Kurucz.

\section{References}

Arnett, W. D. 1988, ApJ, 331, 377

Baade, W. 1926, Astr. Nachr., 228, 359

Baron, E., Branch, D., Hauschildt, P. H., et al. 2000, ApJ, 545, 444

Baron, E., Nugent, P. E., Branch, D., \& Hauschildt, P. H. 2004, ApJ, 616, 91 (B04)

Burrows, A., Hayes, J., \& Fryxell, B. A. 1995, ApJ, 450, 830

Cardelli, Jason A., Clayton, G. C., \& Mathis, J. S. 1988, ApJ, 329, 33

Crowther, P. A., Dessart, L., Hillier, D. J., Abbott, J. B., \& Fullerton, A. W. 2002, A\&A, 392, 653

Dessart L., Crowther P. A., Hillier, D. J., et al. 2000, MNRAS 315, 407

Dessart, L., \& Hillier, D. J. 2005a, A\&A, 437, 667 (Paper I)

Dessart, L., \& Hillier, D. J. 2005b, A\&A, 439, 671 (Paper II)

Eastman, R. G., \& Kirshner, R. P. 1989, ApJ, 347, 771

Eastman, R. G., Schmidt, B. P., \& Kirshner, R. 1996, ApJ, 466, 911

Ensman, L., \& Burrows, A. 1992, ApJ, 393, 742

Hamuy, M., Pinto, P. A., Maza, J., et al. 2001, ApJ, 558, 615 (H01)

Hauschildt, P. H., Best, M., \& Wehrse, R. 1991, A\&A, 247, 21

Hillier, D. J., \& Miller, D. L. 1998, ApJ, 496, 407

Hillier, D. J., \& Miller, D. L. 1999, ApJ, 519, 354

Hirshi, R., Meynet, G., \& Maeder, A. 2004, A\&A, 425, 649

Janka, H. T., \& Müller 1996, A\&A, 306, 167

Jeffery, D. J. 1993, ApJ, 415, 734

Kirshner, R. P., \& Kwan, J. 1974, ApJ, 193, 27

Leonard, C., Filippenko, A. V., Gates, E. L, et al. 2002, PASP, 114, 35 (L02)

Leonard, D. C., Kanbur, S. M., Ngeow, C. C., \& Tanvir, N. R. 2003, ApJ, 594, 247

Li, W. D. 1999, IAU circ. 7294

MacFadyen, A. I., \& Woosley, S. E. 1999, ApJ, 524, 262

MacFadyen, A. I., Woosley, S. E., \& Heger, A. 2001, ApJ, 550, 410

Martins, F., Schaerer, D., Hillier, D. J., \& Heydari-Malayeri, M. 2004, A\&A, 420, 1087

Mitchell, R. C., Baron, E., Branch, D., et al. 2002, ApJ, 574, 293

Najarro, F., Hillier, D. J., \& Stahl, O. 1997, A\&A, 326, 117

Prantzos, N., Doom, C., Arnould, M., \& De Loore, C. 1986, ApJ, 304, 695

Press, W. H., Flannery, B. P., Teukolsky, S. A., \& Vetterling, W. T. 1986, The Art of Scientific Computing (Cambridge University Press)

Schmidt, B. P., Kirshner, R. P., \& Eastman, R. G. 1992, ApJ, 395, 366

Taylor, J. R. 1997, An Introduction to Error Analysis, University Science books, Sausalito, California

Utrobin, V. P., \& Chugai, N. N. 2005, A\&A, 441, 271

Woosley, S. E. 1988, ApJ, 330, 218 
L. Dessart and D. J. Hillier: Type II supernovae spectroscopic modeling, Online Material p 1

\section{Online Material}


L. Dessart and D. J. Hillier: Type II supernovae spectroscopic modeling, Online Material p 2

\section{Appendix A: Errors}

As an accurate distance is the goal of the EPM technique it is worth discussing the error sources, and how they effect the determination of the angular diameter, and hence the distance, in more detail. For simplicity we consider the use of two filters only. Let $F_{i}$ be the observed flux in bandpass $i$, and $F_{i}^{c}$ the reddening corrected flux. Thus

$F_{i}^{c}=F_{i} \exp \left(0.921 R_{i} E(i-j)\right)$

where $R_{i}=A_{i} / E(i-j)$ and $E(i-j)$ is the color excess (e.g., $E(B-V))$. The blackbody color temperature is determined from

$\frac{F_{i}}{F_{j}} \exp (0.921 E(i-j))=\left(\frac{v_{i}}{v_{j}}\right)^{3} \frac{\exp \left(u_{j}\right)-1}{\exp \left(u_{i}\right)-1}$

where $u_{i}=h v_{i} / K T$. Under the assumption that the errors are small, we can determine them using standard techniques (Taylor 1997). Differentiating Eq. (A.2) yields

$\frac{\partial T}{\partial F_{i}}=\frac{T}{F_{i}} \frac{1}{G_{i}-G_{j}}$

and

$\frac{\partial T}{\partial F_{j}}=-\frac{T}{F_{j}} \frac{1}{G_{i}-G_{j}}$

where

$G_{i}=\frac{u_{i}}{1-\exp \left(-u_{i}\right)}$

The expression for the angular diameter, $\theta$ is

$\theta=\left(F_{i} / B_{i}\right)^{1 / 2} \frac{1}{\sqrt{\pi} \xi}$

To allow for the error in $\xi$ we split it into two parts, a random error at fixed temperature, $\sigma_{\xi T}$, and an error attributed to the error in $T$ which can be attributed to an error in $F_{i}, F_{j}$, and $E(i-j)$. Thus, for example,

$\frac{\partial \xi}{\partial F_{i}}=\frac{\partial \xi}{\partial T} \frac{\partial T}{\partial F_{i}}$

and where $\partial \xi / \partial T$ is determined from a set of models.

If we assume the errors are independent we obtain

$$
\begin{aligned}
\sigma_{\theta}= & \frac{\theta}{2}\left(4\left(\frac{\sigma_{\xi T}}{\xi}\right)^{2}\right. \\
& +0.92^{2}\left(R_{i}-\frac{G_{i}}{G_{i}-G_{j}}-\frac{2}{G_{i}-G_{j}} \frac{\partial \ln \xi}{\partial \ln T}\right)^{2} \sigma_{E(i-j)}^{2} \\
& +\left(\frac{G_{j}}{G_{i}-G_{j}}+\frac{2}{G_{i}-G_{j}} \frac{\partial \ln \xi}{\partial \ln T}\right)^{2}\left(\frac{\sigma_{F_{i}}}{F_{i}}\right)^{2} \\
& \left.+\left(\frac{G_{i}}{G_{i}-G_{j}}+\frac{2}{G_{i}-G_{j}} \frac{\partial \ln \xi}{\partial \ln T}\right)^{2}\left(\frac{\sigma_{F_{j}}}{F_{j}}\right)^{2}\right)^{1 / 2} .
\end{aligned}
$$

The value of $\sigma_{\xi T}$ is difficult to determine. An estimate of its value can easily be determined from a grid of models at fixed

\begin{tabular}{|c|c|c|c|}
\hline$T(K)$ & $\overline{G_{i} /\left(G_{i}-G_{j}\right)}$ & $\begin{array}{l}\mathrm{d} \ln \theta / \mathrm{d} \ln F \\
B V\end{array}$ & $\bar{d} \mathrm{~d} \ln \theta / \mathrm{d} E(B-V)$ \\
\hline 6000 & 5.18 & 3.33 & -0.50 \\
\hline 8000 & 5.48 & 3.54 & -0.64 \\
\hline 10000 & 5.89 & 3.83 & -0.83 \\
\hline 12000 & 6.36 & 4.16 & -1.04 \\
\hline 14000 & 6.87 & 4.52 & -1.28 \\
\hline \multicolumn{4}{|c|}{ VI } \\
\hline 6000 & 3.60 & 2.22 & -0.56 \\
\hline 8000 & 3.94 & 2.46 & -0.75 \\
\hline 10000 & 4.35 & 2.74 & -0.97 \\
\hline 12000 & 4.78 & 3.05 & -1.21 \\
\hline 14000 & 5.24 & 3.37 & -1.47 \\
\hline \multicolumn{4}{|c|}{$\mathrm{BI}$} \\
\hline 6000 & 2.40 & 1.39 & -0.54 \\
\hline 8000 & 2.57 & 1.50 & -0.71 \\
\hline 10000 & 2.77 & 1.64 & -0.92 \\
\hline 12000 & 3.00 & 1.80 & -1.15 \\
\hline 14000 & 3.24 & 1.97 & -1.39 \\
\hline
\end{tabular}
effective temperature encompassing a range of $\mathrm{SN}$ type models (e.g., density exponent). However, there is no guarantee that
Table A.1. Errors in $\theta$ due to flux and reddening errors.

this grid is representative of true SN. A further source of error is the fact that models do not exactly match the observations, and this will contribute an error greater than those introduced by the dispersion in SN properties, This error could, if known, be incorporated into $\sigma_{\xi T}$.

In Table A.1, we list $G_{i} /\left(G_{i}-G_{j}\right), \mathrm{d} \ln \theta / \mathrm{d} \ln F$, $\mathrm{d} \ln \theta / \mathrm{d} E(B-V)$ for three different passband combinations. We ignore the coupling with $\xi$, and assume that the fractional errors in the two pass bands are identical. As readily apparent, the fractional error in $\theta$ due to an error in flux increases with $\mathrm{SN}$ temperature - this is simply due to the increasing insensitivity of the blackbody slope to temperature as we approach the Rayleigh-Jeans regime. We also note that a $1 \%$ error in flux is generally more important than a 0.01 error in $E(B-V)$. This somewhat surprising result occurs since the effect of the reddening on the temperature estimate, and the direct effect of reddening on the angular diameter (because of a change in flux) partially compensate. This has been previously noted by Leonard et al. (2002), Eastman et al. (1996), and Schmidt et al. (1992). Not surprisingly, we also see that the BI passband combination, with its wider wavelength coverage, gives potentially the smallest errors. Finally we note that in the $B V$ band, the angular diameter is very sensitive to flux errors. A random error of $3 \%$ in each passband leads to a 10 to $14 \%$ error in $\theta$.

Errors associated with $\xi$ are specific to the EPM technique. However the other error sources will also apply, in a complicated fashion, to the SEAM technique. The primary distinction is that the SEAM technique uses global fitting, whereas the EPM is sensitive to local errors in 2 or 3 pass bands. These errors arise, for example, from errors in the abundance, the neglect of some species, from observational errors, and from inadequacies in the model. 
L. Dessart and D. J. Hillier: Type II supernovae spectroscopic modeling, Online Material p 3

\section{Appendix B: Distance determination}

The relation between the distance and $\theta$ is

$$
\frac{\theta}{v}=\frac{\left(t-t_{\mathrm{exp}}\right)}{D} \text {. }
$$

Several different approaches can be used to estimate $D$ and $t_{\text {exp }}$. Because $t$ is known accurately, we must use $t$ as the independent variable. In standard form the equation is

$$
y=a+b t
$$

If we use least squares, errors for $a$ and $b$ can be readily determined from the accuracy of the fit. Thus we have $D=1 / b$ and

$\sigma_{D} / D=\sigma_{b} / b$

Similarly, $t_{\exp }=-a / b$ and

$\sigma_{t_{\mathrm{exp}}}=\left(\sigma_{a}^{2}+t_{\mathrm{exp}}^{2} \sigma_{b}^{2}-2 t_{\mathrm{exp}} \sigma_{a b}\right)^{1 / 2} / b$

where $\sigma_{a b}$ is the covariance of $a$ and $b$.
The errors on $\theta / v$ are difficult to determine accurately. However, in general we expect the errors in $\theta / v$ to scale with $\theta / v$. For simplicity, we therefore perform weighted least squares fitting assuming constant fractional errors. Closer examination of the error analysis discussed earlier suggests that the fractional errors in $\theta / v$ could be somewhat larger at earlier epochs, but given the additional influence of systematic errors, we have made no effort to take this into account.

We also used robust fitting techniques (see Press et al. 1986) with weights, but the answers were generally similar (i.e., within 1 sigma) to those obtained with least squares fitting. As a test, we also tried fitting the data assuming constant errors. However, this gives too much weight to the data of day 38 , and leads to unrealistically small error bars.

The errors on $D$ and $t_{\exp }$ listed in the tables are from the fit only, and thus are a MINIMUM estimate of the error. No systematic errors, which are difficult to determine, have been taken into account. Systematic errors will arise from errors in reddening, and possibly, errors in the model. It is also likely, for example, that errors in adjacent $\theta / v$ estimates are correlated, since the spectra are similar, and hence model errors are likely to be similar. 